\title{
Analysis of S. pombe SIN protein association to the SPB reveals two genetically separable states of the SIN
}

\author{
Paulina Wachowicz' ${ }^{1}$, Anastasia Chasapi ${ }^{2}$, Andrea Krapp ${ }^{1}$, Elena Cano del Rosario ${ }^{1}$, Daniel Schmitter ${ }^{3}$, \\ Daniel Sage ${ }^{3}$, Michael Unser ${ }^{3}$, loannis Xenarios ${ }^{2}$, Jacques Rougemont ${ }^{4}$ and Viesturs Simanis ${ }^{1, *}$
}

\begin{abstract}
The Schizosaccharomyces pombe septation initiation network (SIN) regulates cytokinesis, and asymmetric association of SIN proteins with the mitotic spindle pole bodies (SPBs) is important for its regulation. Here, we have used semi-automated image analysis to study SIN proteins in large numbers of wild-type and mutant cells. Our principal conclusions are: first, that the association of Cdc7p with the SPBs in early mitosis is frequently asymmetric, with a bias in favour of the new SPB; second, that the early association of Cdc7p-GFP to the SPB depends on Plo1p but not Spg1p, and is unaffected by mutations that influence its asymmetry in anaphase; third, that Cdc7p asymmetry in anaphase B is delayed by Pom1p and by activation of the spindle assembly checkpoint, and is promoted by Rad24p; and fourth, that the length of the spindle, expressed as a fraction of the length of the cell, at which Cdc7p becomes asymmetric is similar in cells dividing at different sizes. These data reveal that multiple regulatory mechanisms control the SIN in mitosis and lead us to propose a two-state model to describe the SIN.
\end{abstract}

KEY WORDS: S. pombe, Cytokinesis, SIN

\section{INTRODUCTION}

Asymmetric events are of fundamental importance in biology. Asymmetry of centrosome behaviour and inheritance is also implicated in an increasing number of cellular and developmental processes (Knoblich, 2010; Tajbakhsh et al., 2009). Schizosaccharomyces pombe is an excellent model for the analysis of the conserved basic mechanisms of cell division. $S$. pombe cells are rod-shaped, grow by tip-elongation and divide by binary fission. As in higher eukaryotes, a contractile actomyosin ring (CAR) is important for cytokinesis (reviewed by Ishiguro, 1998; Pollard and Wu, 2010) and acts as a 'guide' for synthesis of the division septum (Proctor et al., 2012). The position of the division plane is determined by signalling from the nucleus and the cell tips (reviewed by Goyal et al., 2011; Oliferenko et al., 2009). The coordination of cytokinesis with other mitotic events is assured by a conserved network of protein kinases known as the septation initiation network (SIN). The SIN is one of two

${ }^{1}$ Cell cycle control laboratory, Ecole Polytechnique Fédérale de Lausanne (EPFL), SV-ISREC, 1015 Lausanne, Switzerland. ${ }^{2}$ Swiss-Prot. Group and Vital-IT Group, Swiss Institute of Bioinformatics (SIB), 1015 Lausanne, Switzerland. ${ }^{3}$ Biomedical Imaging Group, Ecole Polytechnique Fédérale de Lausanne (EPFL), 1015 Lausanne, Switzerland. ${ }^{4}$ Bioinformatics and Biostatistics Core Facility, Ecole

Polytechnique Fédérale de Lausanne (EPFL), 1015 Lausanne, Switzerland.

*Author for correspondence (viesturs.simanis@epfl.ch)

Received 18 July 2014; Accepted 8 December 2014 conserved nuclear Dbf2-related (NDR) kinase signalling pathways in $S$. pombe, the other being the morphology network (MOR). As in other eukaryotes, they control cell proliferation and growth (reviewed by Gupta and McCollum, 2011). Loss of SIN signalling causes failure of cytokinesis, generating multinucleated cells (Nurse et al., 1976). In contrast, continuous SIN signalling results in multiseptated cells (Minet et al., 1979). Ectopic activation of SIN signalling induces CAR and septum formation during any stage of the cell cycle (Schmidt et al., 1997), emphasising the importance of coordinating cytokinesis with mitosis.

The SIN plays multiple roles during cytokinesis (reviewed by Goyal et al., 2011; Johnson et al., 2012; Roberts-Galbraith and Gould, 2008) and mitotic commitment (Grallert et al., 2012). The spindle pole body (SPB) serves as a microtubule-organising centre, and coordination point for cell cycle regulators (Grallert et al., 2012; Grallert et al., 2013; Hagan, 2008). Association of SIN proteins to the SPB plays an important part in the regulation of the SIN (reviewed by Johnson et al., 2012; Simanis, 2003). SIN proteins associate with a tripartite scaffold (Cdc11p-Sid4pPpc89p), which is essential for signalling (Chang and Gould, 2000; Krapp et al., 2001; Morrell et al., 2004; Rosenberg et al., 2006; Tomlin et al., 2002). The core SIN components are three protein kinases (Cdc7p, Sid1p and $\operatorname{Sid} 2 \mathrm{p}$ ), and their regulatory subunits (Spg1p, Cdc14p and Mob1p, respectively) (Fankhauser and Simanis, 1993; Fankhauser and Simanis, 1994; Guertin et al., 2000; Hou et al., 2000; Salimova et al., 2000; Schmidt et al., 1997; Sparks et al., 1999). Signalling is mediated by the small GTPase Spg1p, which is regulated by the GTPase-activating protein (GAP) Cdc16p that interacts with Spg1p through the scaffold Byr4p, (Fankhauser et al., 1993; Furge et al., 1998; Minet et al., 1979; Song et al., 1996), Etd1p (Daga et al., 2005; García-Cortés and McCollum, 2009; Lahoz et al., 2010) and the conserved kinase Plo1p (Rachfall et al., 2014; Tanaka et al., 2001). Immunoelectron microscopy has shown that Ppc89p, Sid4p (Rosenberg et al., 2006) and Sid2p (Sparks et al., 1999) are localized at the cytoplasmic side of the SPB, suggesting that SIN signalling is activated in the cytoplasm.

The intensity of some SIN protein SPB-associated signals changes during mitosis (Cerutti and Simanis, 1999; Feoktistova et al., 2012; García-Cortés and McCollum, 2009; Simanis, 2003; Sohrmann et al., 1998; Wu and Pollard, 2005). In anaphase B, Cdc7p (Grallert et al., 2004; Sohrmann et al., 1998) and Sid1p (Guertin et al., 2000) associate with the new SPB (nSPB), whereas Byr4p and Cdc16p associate with the old SPB (oSPB) (Cerutti and Simanis, 1999; Li et al., 2000). Mutants that compromise asymmetric distribution of SIN proteins on the SPBs during mitosis deregulate septation (García-Cortés and McCollum, 2009; Singh et al., 2011; Sohrmann et al., 1998). If 
the SIN fails to signal, then SIN protein asymmetry in anaphase is not established. Therefore, it has been proposed that there is a feedback loop within the SIN, mediated by Sid2p and that phosphorylation of the scaffold protein Cdc11p by Sid $2 p$ contributes to this regulation (Bajpai et al., 2013; Feoktistova et al., 2012; Johnson et al., 2012).

The data presented in this study suggest there are at least two, genetically separable, states of the SIN; an early state that is dependent upon Plolp for its establishment, and a late state, which is dependent upon Spg1p.

\section{RESULTS}

The RodCellJ ImageJ plugin (Schmitter et al., 2013) was used to examine large numbers of mitotic $S$. pombe cells. The cells express a tagged SIN protein together with mCherry-tagged Pcplp (PcplpCHY hereafter) to visualise SPBs, and the kinetochore marker mCherry-tagged Cnplp as required (Cnplp-CHY hereafter) (Alvarez-Tabarés et al., 2007); the different intensity of the Cnplp-CHY and Pcplp-CHY signals allows them to be differentiated in early mitosis. To facilitate comparison between cells of different size, the data are presented as the ratio of the SPB intensities plotted against the separation of the SPBs, expressed as a fraction of the cell length (length fraction; LF; Fig. 1C). The raw data were divided into ten bins with a step size of LF0.1. To facilitate the analysis and presentation of the data, three states were defined; symmetry as $\leq 2$-fold difference between the GFP signals on the two SPBs; transition as $>2$-fold but $<4$-fold; asymmetric as $\geq 4$-fold difference.
A

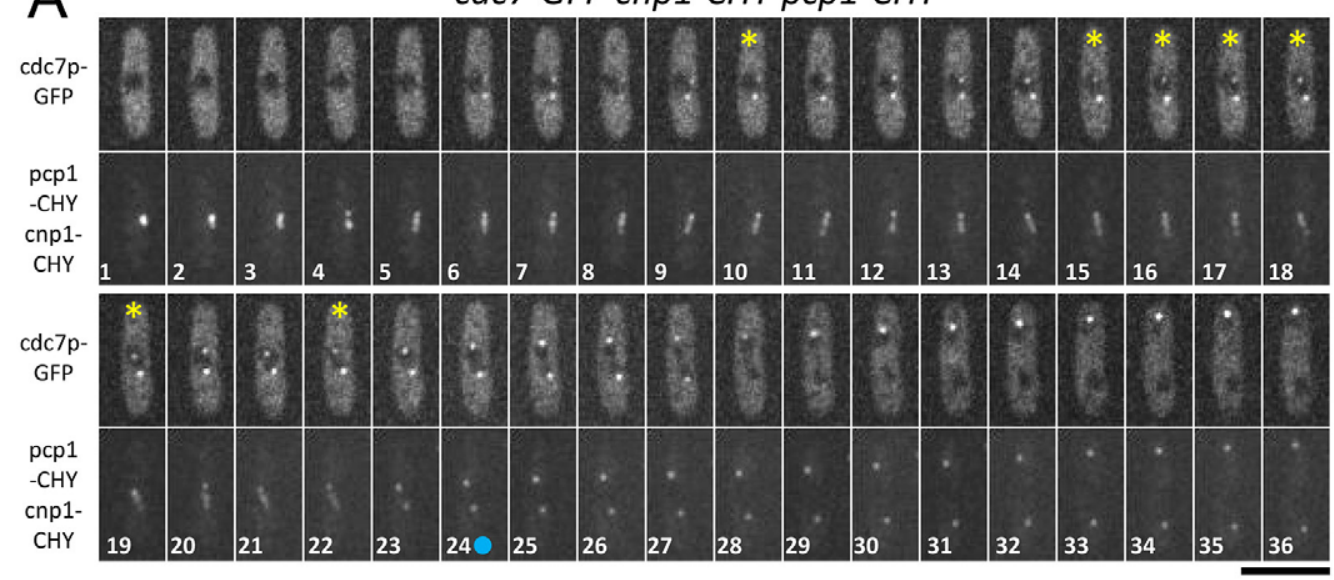

B

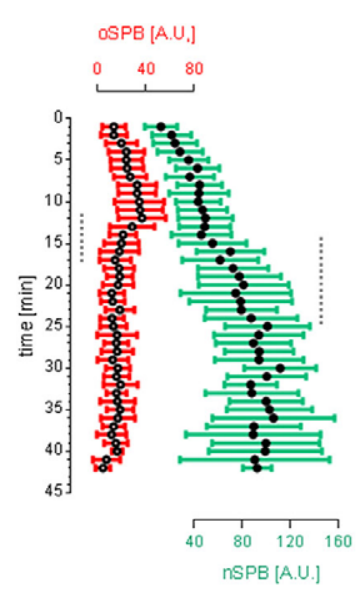

D

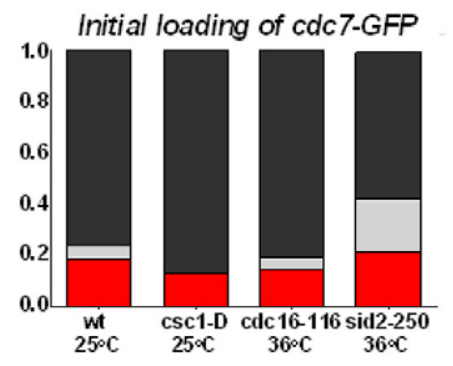

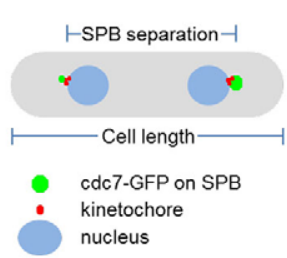

E

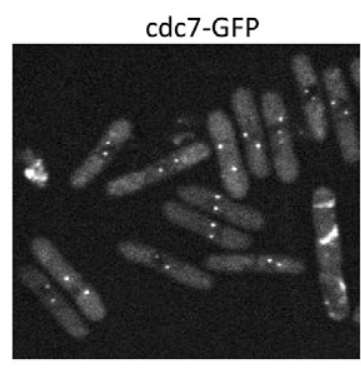

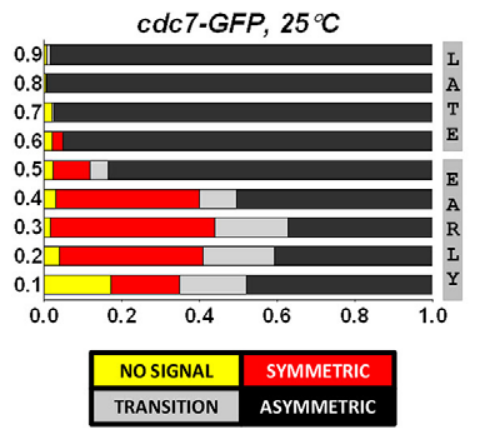

TRANSITION ASYMMETRIC

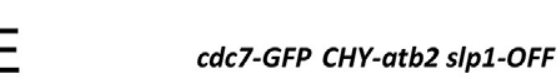




\section{SIN protein association with the SPB in mitosis in wild-type cells \\ Sid4p-GFP, Cdc11p-GFP, Spg1p-GFP and Cdc7p-GFP}

The SPB-associated signals of Cdc11p-GFP (supplementary material Fig. S1A) and Sid4p-GFP (supplementary material Fig. S1B) were symmetric in the vast majority of cells throughout mitosis, as described previously (Chang and Gould, 2000; Krapp et al., 2001). Spg1p-GFP is symmetrical in the majority of cells throughout mitosis (supplementary material Fig. S1C); its intensity increased 1.5 -fold at both SPBs during mitosis (supplementary material Fig. S1D,E). Cdc11p-GFP showed a similar increase, whereas Sid4p-GFP remained constant (data not shown). This is consistent with the slow turnover of Sid4p-GFP (Feoktistova et al., 2012) and indicates that the asymmetry of SIN proteins is not due to changes in the level of scaffold proteins at the SPBs.

The essential effector of Spg1p, Cdc7p (Schmidt et al., 1997), was chosen as the primary reporter of SIN protein behaviour. It associates with both SPBs in early mitosis, then only with the nSPB during anaphase B (Grallert et al., 2004; Sohrmann et al., 1998) (Fig. 1A).

As cells progressed through mitosis, the Cdc7p-GFP signal at the oSPB decreased, whereas the nSPB brightened (Fig. 1B), consistent with previous studies (Cerutti and Simanis, 1999; García-Cortés and McCollum, 2009). The rate was similar at both SPBs $\left(3.6 \pm 0.5 \mathrm{AU} \mathrm{min}^{-1}\right.$ for the $\mathrm{nSPB}$ and $-4.1 \pm$ 0.6 AU min ${ }^{-1}$ at the oSPB). The signal at the oSPB began to decline $2 \mathrm{~min}$ before the nSPB brightened, suggesting that asymmetry initiates at the oSPB.

In wild-type cells, the fraction of asymmetric Cdc $7 p-G F P$ signals increased with SPB separation (Fig. 1C). At LF0.6 $(\approx 8.5 \mu \mathrm{m}$ spindle in a $14 \mu \mathrm{m}$ wild-type cell $)>95 \%$ of cells showed an asymmetric signal, rising to $>99 \%$ at LF0.8 $(\approx 11.5 \mu \mathrm{m}$ spindle in wild-type). These data are consistent with previous analysis of wild-type cells (Feoktistova et al., 2012).

\section{SPB association of Cdc7p-GFP in early mitosis is asymmetric}

Filming revealed an initial asymmetric SPB association of Cdc7p-GFP in 25 of 33 cells (76\%); two cells (6\%) had signals on both SPBs, with asymmetric intensity, and six cells (18\%) showed a symmetric signal. Cdc7p-GFP associated with the nSPB first in 21/27 cells (78\%) (Fig. 1D). Examples of Cdc7pGFP loading first to the nSPB (supplementary material Fig. S1F) and oSPB (Fig. 1A) are presented. Thus, the initial association of Cdc7p-GFP with SPBs is asymmetric in $\approx 80 \%$ of cells, with a $\approx 3: 1$ bias towards the nSPB.

These films also revealed transient fluctuations in the relative intensity of the SPB-associated Cdc7p-GFP signal in some (Fig. 1A) but not all cells (supplementary material Fig. S1F), which might explain why $\sim 40 \%$ of cells in single timepoint images showed a significantly asymmetric signal in early mitosis (Fig. 1C; LF0.2 to 0.3, corresponding to SPB separation of $\sim 3-$ $4 \mu \mathrm{m}$ in a $14 \mu \mathrm{m}$ wild-type cell). This might not have been observed previously because they were performed by indirect immunofluorescence (Sohrmann et al., 1998).

Given that Cdc7p-GFP asymmetry occurs in anaphase, a conditionally expressed allele of slpl (also known as CDC20) (Petrova et al., 2013) was used to investigate whether SPB association of $\mathrm{Cdc} 7 \mathrm{p}-\mathrm{GFP}$ requires the anaphase-promoting complex/cyclosome containing Slp1p $\left(\mathrm{APC} / \mathrm{C}^{\mathrm{Slp} 1 \mathrm{p}}\right)$. Expression of $\operatorname{slp1}$ was silenced in the strain atb2-CHY $c d c 7-G F P$ (hereafter referred to as slp1-OFF). Cdc7p-GFP associated with both SPBs in $95 \%$ of the arrested cells ( 240 of 253 cells; Fig. 1E); $85 \%$ of these showed a symmetric signal (LF0.1 and 0.2; data not shown). Given that the initial SPB association of Cdc7p-GFP is often asymmetric, the difference between these data and those in Fig. 1C might have arisen because the slpl-OFF cells are arrested for an extended period in metaphase, giving sufficient time for the Cdc $7 \mathrm{p}-\mathrm{GFP}$ to equalise on the SPBs.

\section{The initial SPB association of Cdc7p-GFP is not influenced by mutants affecting its anaphase $B$ asymmetry}

The STRIPAK-related SIN-inhibitory phosphatase complex (SIP) is required to establish Cdc7p-GFP and GFP-Sid1p asymmetry in anaphase (Singh et al., 2011). The SPB association of Cdc7pGFP in early mitosis was asymmetric in seven of eight $\csc 1-D$ cells examined (Fig. 1D). Cdc7p-GFP remained symmetric in the majority of cells in the later stages of mitosis, as expected (Fig. 2C), although a minority of cells displayed a Cdc7p-GFP signal that differed by $>4$-fold. Consistent with earlier studies (Singh et al., 2011), the signal intensity increased at both SPBs as cells progressed through mitosis (data not shown).

As described previously (Schmidt et al., 1997; Sohrmann et al., 1998), association of Cdc7p-GFP with the SPBs in anaphase was mostly symmetric in cdc16-116 (compare Fig. 2B,D). As cells entered mitosis 17 of 21 cdc16-116 cells (79\%) showed an asymmetric SPB association of Cdc7p-GFP with the SPBs, one cell $(5 \%)$ showed association with both SPBs, with a stronger signal on one pole than the other, and three cells (14\%) showed a symmetric signal (Fig. 1D). Therefore, despite the reduction of GAP function, the initial association of Cdc7p-GFP with the SPBs was asymmetric in $>80 \%$ of the cells. No Cdc $7 p-$ GFP signal was observed prior to SPB separation (data not shown). Given that the Cdc7p-GFP signal is symmetric in the majority of $c d c 16-116$ cells in anaphase compared to Cdc $7 \mathrm{p}-\mathrm{GFP}$ in wild-type cells at $36^{\circ} \mathrm{C}$, it was not possible to determine which SPB initially loaded Cdc $7 \mathrm{p}-$ GFP. The Cdc7p-GFP signal reached maximum intensity more rapidly in cdc16-116 than in wild-type cells (Fig. 2G; LF0.1-0.4).

The early loading of Cdc7p-GFP was still asymmetric in sid2250 cells at $36^{\circ} \mathrm{C}$ (eight of 14 cells), indicating that $\mathrm{Sid} 2 \mathrm{p}$ is not obligatory for the asymmetric early loading of Cdc7p-GFP (Fig. 1D). Consistent with previous studies (Feoktistova et al., 2012; Mishra et al., 2005), Cdc7p-GFP remained largely symmetric in sid2-250 anaphase cells both at $25^{\circ} \mathrm{C}$ (Fig. 2E) and $36^{\circ} \mathrm{C}$ (Fig. 2F). The intensity increased at both SPBs until LF0.3 and then plateaued (Fig. 2H). Taken together, these data are consistent with the idea that the association of $\mathrm{Cdc} 7 \mathrm{p}$ with the SPB early in mitosis is not affected by the regulators of its asymmetric distribution in anaphase. However, although the cscl$D$ mutant is a null allele, sid2-250 and cdc16-116 are 'tight' conditional alleles. Thus, we cannot formally exclude the possibility that the latter mutants have residual activity that still permits normal loading of Cdc7p-GFP at mitotic onset. Future analysis of null mutants will be necessary to examine this.

\section{Spg1 is not required for Cdc7p association with the SPB in early mitosis}

Inactivation of Spg1p revealed that $\mathrm{Cdc} 7 \mathrm{p}-\mathrm{GFP}$ was still associated with the SPB in early mitosis in $\operatorname{spg} 1-B 8$ at $36{ }^{\circ} \mathrm{C}$ (compare Fig. 2I with Fig. 2B). In late mitosis, the intensity of the signal at the nSPB became fainter, rather than brighter (Fig. $2 \mathrm{H}$ ), and the signal remained symmetrical in $>30 \%$ of cells (Fig. 2I). Cytokinesis failed in the mutant cells, indicating that 

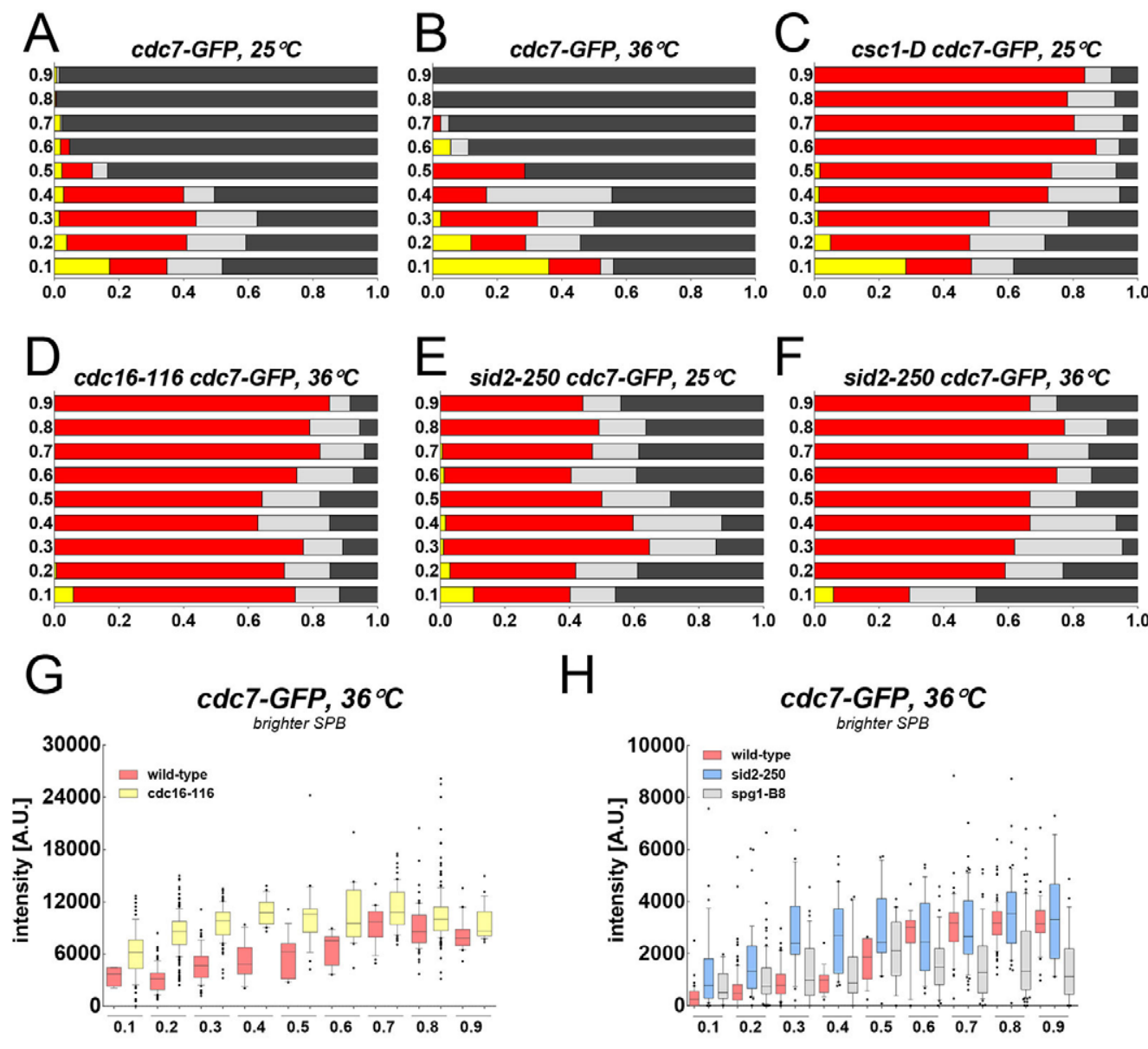

Fig. 2. Analysis of Cdc7p-GFP distribution in wild-type and mutant cells. Cells of the indicated strains were synchronised and analysed at the indicated temperatures as described in the Materials and Methods.

(A-F, I) Analysis of Cdc7p-GFP SPB localisation presented as described for Fig. 1C. A is identical to Fig. 1B, and is re-presented here for convenience. $(\mathrm{G}, \mathrm{H})$ Box plots of the intensity of Cdc7p-GFP at the brighter SPB. Cells were synchronised and samples were taken for analysis as they progressed through mitosis. The intensity (A.U., arbitrary units) is plotted on the ordinate, LF on the abscissa. The box shows $25-75 \%$ range for the population, the line indicates the median. The bars indicate $10 \%$ and $90 \%$ range for the population, and dots indicate more extreme individual values.
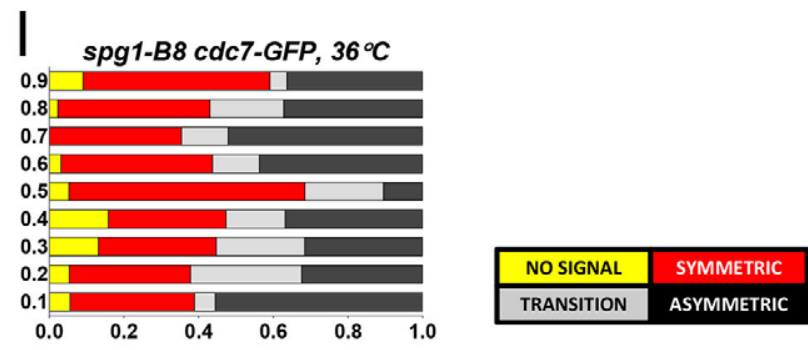

Spglp had been inactivated (data not shown). If cells were incubated for $5 \mathrm{~h}$ at $36^{\circ} \mathrm{C}$ before analysis, we did not observe any SPB-associated Cdc7p-GFP signal (data not shown), consistent with previous studies (Sohrmann et al., 1998). Thus, in the first cycle after inactivation of Spg1p, the early mitotic association of Cdc7p-GFP with the SPB is independent of Spg1p, and the late mitotic association and transition to asymmetry requires $\mathrm{Spg} 1 \mathrm{p}$ function.

The kinase POLO is required for Cdc7p-GFP SPB association in early mitosis but not in anaphase

The conserved POLO-like kinase Plo1p has multiple roles during mitosis (Bähler et al., 1998; Ohkura et al., 1995). Epistasis analysis places plo1 upstream of the SIN (Tanaka et al., 2001); Plo1-ts4 is a hypomorphic allele whose primary defect is in SIN regulation. There was no detectable Cdc $7 \mathrm{p}-\mathrm{GFP}$ signal at the SPBs in a large fraction of early mitotic plol-ts 4 cells at $36^{\circ} \mathrm{C}$ [Fig. 3A,B; 79\% of LF0.1 cells $(n=279)$, and $73 \%$ of LF0.2 $(n=193)]$; when a signal was observed, it was asymmetric. This was recapitulated in the plo1-as3 mutant (Grallert et al., 2012) (data not shown). Mixing of early mitotic $c d c 7-G F P$ and $c d c 7-$ GFP plo1-ts 4 cells at $36^{\circ} \mathrm{C}$ showed that Cdc7p-GFP was detectable in wild-type cells at $36^{\circ} \mathrm{C}$ (Fig. 3C). However, by LF0.6, more than $80 \%$ of cells had an asymmetric signal with Cdc $7 \mathrm{p}-\mathrm{GFP}$ on one SPB in anaphase B (compare Fig. 3B with Fig. 2B). The signal intensity in anaphase was comparable to wild-type (Fig. 3D). Thus, Plolp is required for the SPB recruitment of $\mathrm{Cdc} 7 \mathrm{p}-\mathrm{GFP}$ at the onset of mitosis. Furthermore, the appearance of $\mathrm{Cdc} 7 \mathrm{p}$ at one SPB in anaphase B does not require $\mathrm{SPB}$ association of $\mathrm{Cdc} 7 \mathrm{p}$ earlier in mitosis.

\section{Symmetric SPB association of Mob1p-GFP in early mitosis requires Plo1p}

The Mob1p-Sid2p complex (Hou et al., 2000; Salimova et al., 2000) was examined using mob1-GFP (Salimova et al., 2000), because sid2-GFP (Sparks et al., 1999) is functionally compromised 


\section{A}

cdc7p-GFP

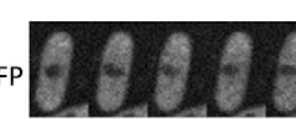

plo1-ts4 cdc7-GFP pcp1-CHY cnp1-CHY, $36^{\circ} \mathrm{C}$
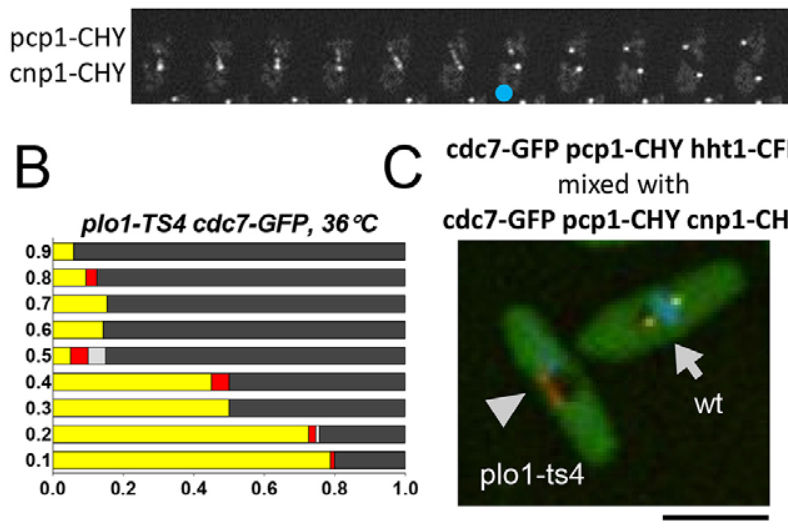

\section{$E$}
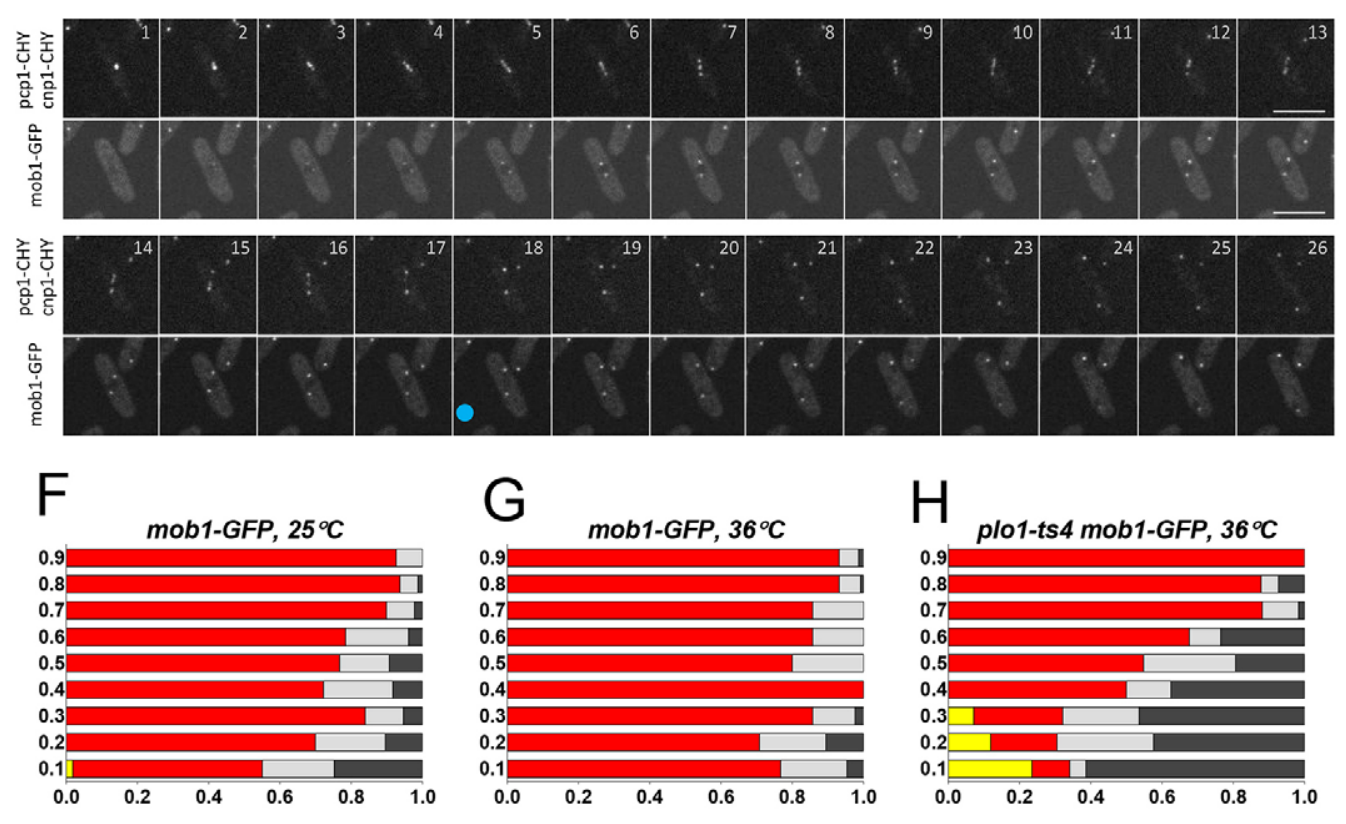

(Grallert et al., 2012; Salimova et al., 2000). Mob1p-GFP associated with both SPBs in most cells, exceeding $98 \%$ by LF0.7 (Fig. 3F,G), consistent with previous observations (Hou et al., 2000; Salimova et al., 2000). Filming of Mob1-GFP revealed some fluctuations in the relative intensity of the two SPBs throughout mitosis (Fig. 3E). In the early stages of mitosis, the Mob1p-GFP signal was seen on both SPBs in $\approx 75 \%$ of cells and on one SPB in $\approx 25 \%$ cells. This was not noted previously (Hou et al., 2000; Salimova et al., 2000), and is most likely due to improvements in detection. Reduction of Plo1p activity increased the fraction of cells with asymmetric and transition Mob1p-GFP early in mitosis (LF0.1-0.4) compared to wild-type (compare Fig. $3 \mathrm{H}$ to Fig. 3G). Furthermore, no signal was detected in $\sim 25 \%$ of LF0.1 cells, compared to $2 \%$ in wild-type. However, in late anaphase cells ( $\mathrm{LF}>0.8$ ), the fraction of cells with a symmetrical signal was similar to wild-type. Thus, decreased plo1
Fig. 3. Analysis of SIN proteins in mitosis and effect of loss of plo1 function. The marked strains were analysed at the indicated

temperature. The graphs in $\mathrm{B}$ and $\mathrm{F}-$ $\mathrm{H}$ are presented as described for Fig. 1C. (A) Localisation of Cdc7pGFP in plo1-ts 4 cells. Cells were synchronised and images were taken every minute as cells progressed through mitosis. The blue dot indicates anaphase onset. Note the absence of Cdc7p-GFP in early mitosis, followed by the asymmetric appearance of Cdc7p-GFP in anaphase B. (B) Analysis of Cdc7pGFP in plo1-ts 4 at $36^{\circ} \mathrm{C}$; the wild-type control is shown in Fig. 2B. (C) plo1ts 4 cdc7-GFP pcp1-CHY cnp1-CHY cells were mixed with plo1 ${ }^{+}$cdc7GFP pcp1-CHY hht1-CFP cells, synchronised, incubated at $36^{\circ} \mathrm{C}$ and images were taken in mitosis. Note the presence of a Cdc7p-GFP signal on the SPBs of the plo $1^{+}$cell (blue nuclear signal) and its absence from the plo1-ts 4 cell. (D) Box plot of the Cdc7p-GFP signal in the indicated mutants presented as described in Fig. 2G. (E) Analysis of Mob1p-GFP. Cells were synchronised and images were taken every minute as cells progressed through mitosis. Note the transient fluctuation of signal intensity in frames 11, 23 and 24. The blue dot indicates anaphase onset. $(\mathrm{F}-\mathrm{H})$ The distribution of Mob1p-GFP was analysed in the indicated strains. Scale bars: $10 \mu \mathrm{m}$.

activity affects the early, but not the late mitotic SPB association of Mob1p-GFP, as observed for Cdc7p-GFP.

The association of GFP-Sid1p with the SPB is spatially unstable in early mitosis

Approximately $90 \%$ of cells had an asymmetric GFP-Sid1p signal at the SPB at LF0.6, rising to $>98 \%$ at LF0.7 (Fig. 4A-C), consistent with previous studies (Guertin et al., 2000). However, $>50 \%$ of cells with short spindles (LF0.1-0.3) displayed an SPBassociated GFP-Sid1p signal (Fig. 4B,C). Filming revealed that the association of GFP-Sid1p with the SPB was spatially unstable in early mitosis, with a signal seen at one SPB, both SPBs or not at all, in sequential frames (Fig. 4A), before stabilising at the nSPB in anaphase B (note that 'stable' in this context indicates a fixed location for the protein). Faint, symmetric signals in early 


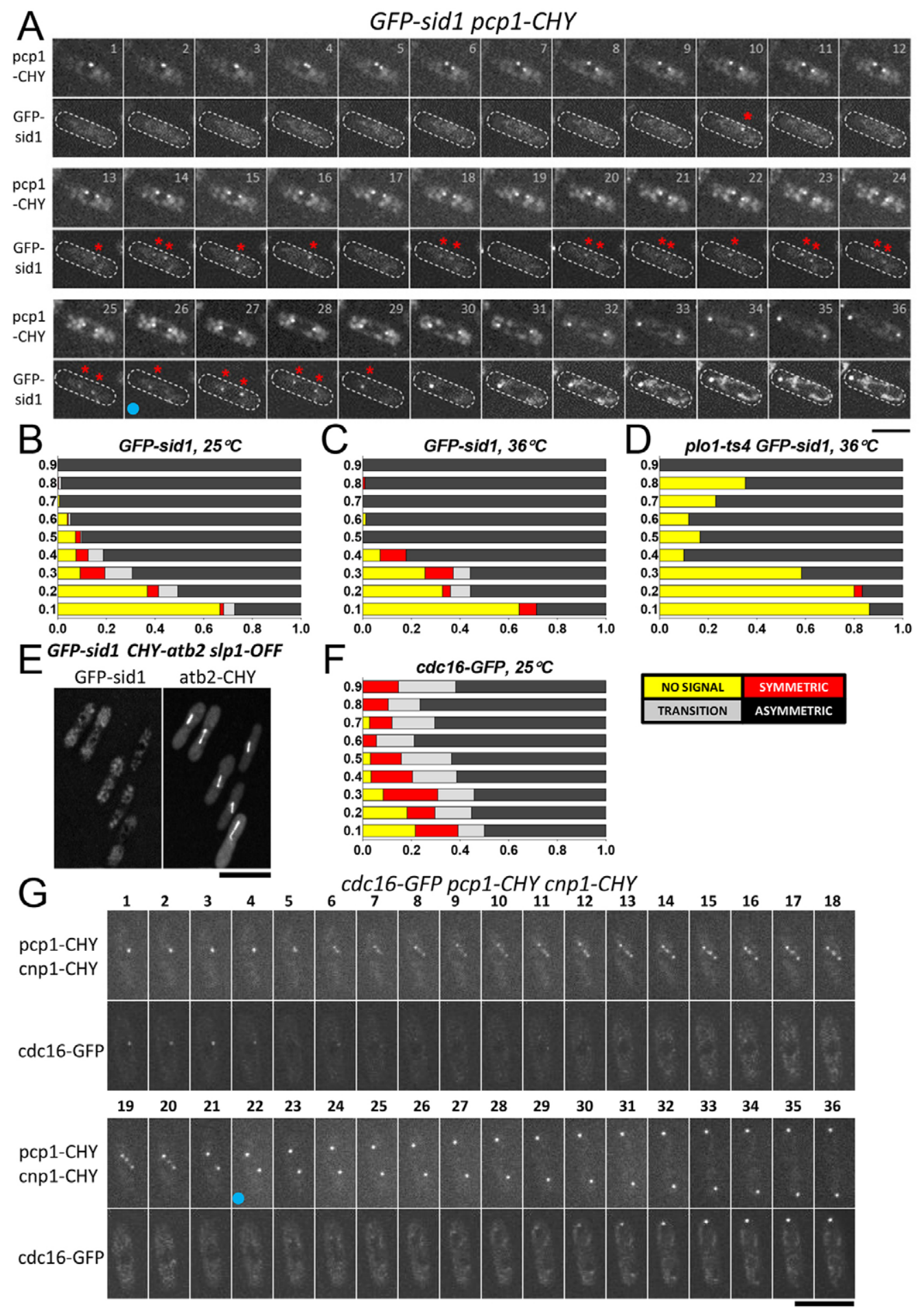

Fig. 4. Analysis of GFP-Sid1p and Cdc16p-GFP. (A) Localisation of GFP-Sid1p during mitosis. Cells were synchronised and images were taken every minute as cells progressed through mitosis. The blue dot indicates anaphase B onset. In frames 10-29, the red asterisks indicate the presence of GFP-Sid1p on the SPB. (B-D) Cells were synchronised and the intensity of GFP-Sid1p was analysed. The graphs are presented as described for Fig. 1C. (E) The indicated strain was arrested as described in the Materials and Methods. Note the absence of GFP-Sid1p from the SPB. (F) The indicated strain was synchronised and the intensity of Cdc16p-GFP was analysed. The graph is presented as described for Fig. 1C. (G) Localisation of Cdc16GFP. Cells were synchronised and images were taken every minute as cells progressed through mitosis. The blue dot indicates anaphase B onset. Scale bars: $10 \mu \mathrm{m}$.

pcp1-CHY cnp1-CHY

mitotic cells, although not an unstable SPB association, have been noted previously (Guertin et al., 2000). Inactivation of Plolp decreased the number of cells with a GFP-Sid1p signal in early mitosis $(\mathrm{LF}<0.4)$, and increased the number of cells without a detectable signal in anaphase (Fig. 4D; supplementary material Fig. S2A). GFP-Sid1p was absent from the SPBs in most slp1$O F F$ cells [Fig. 4E; no signal in $93 \%$ of cells $(n=269)$; signal on one SPB in $2 \%$ of cells; signal on both SPBs in $5 \%$ of cells]. This demonstrates that the $\mathrm{APC} / \mathrm{C}^{\mathrm{Slp} 1 \mathrm{p}}$ must function for stable recruitment of GFP-Sid1p to the SPB.

\section{The GAP Byr4p-Cdc16p}

The signal of Cdc16p-GFP became very faint or undetectable on both SPBs shortly after SPB separation (Fig. 4G), reappearing on the oSPB in anaphase, as described previously (Cerutti and Simanis, 1999). A significant fraction of cells in early mitosis had no detectable Cdc16p-GFP signal. The fraction of cells with an asymmetric signal increased as cells progressed through mitosis (Fig. 4F).

Filming of GFP-byr4 cells revealed that it remained associated with the SPBs early in mitosis, then became faint or undetectable 
and subsequently reappeared at the oSPB (Fig. 5A). Some spatial instability of the GFP-Byr4p signal was seen as the signal declined. In $\mathrm{LF}<0.1$ cells, $52 \%$ of signals were symmetric, $19 \%$ were transition and $26 \%$ were asymmetric; no signal was observed in $3 \%$ of cells. No signal was detected in 18 and $15 \%$ of LF0.2 and LF0.3 cells, respectively (Fig. 5B). Cells with longer spindles (Fig. $5 \mathrm{~B} ; \quad \mathrm{LF} \geq 0.6$ ) showed predominantly asymmetric signals, as described previously (Cerutti and Simanis, 1999; Li et al., 2000), and the fraction of cells with no detectable signal decreased to $<2 \%$.

Surprisingly, given that Byr4p and Cdc16p are interdependent for localisation (Cerutti and Simanis, 1999), the numbers of asymmetric cells did not match those observed for GFP-Cdc16p in anaphase. This discrepancy might arise because the $c d c 16$ GFP allele is partially compromised (the $c d c 16-G F P$ cdc16-D strain becomes multiseptated at $>34^{\circ} \mathrm{C}$; data not shown).

GFP-Byr4p was associated with both SPBs in $95 \%$ of metaphase-arrested slp1-OFF cells (Fig. 5C; $n=111$ ), which is consistent with localisation of Byr4p by indirect immunofluorescence in nda3-KM311 arrested cells (Krapp et al., 2003). The intensity of the GFP-Byr4p signal in the arrested cells was significantly lower than that seen in late anaphase (Fig. 5D; $P<0.001$ ). Taken together, these data indicate that the removal of Byr4p from the SPBs requires $\mathrm{APC} / \mathrm{C}^{\mathrm{Slp} 1 \mathrm{p}}$ function. Comparison with Cdc7p-GFP showed that GFP-Byr4p becomes asymmetric significantly before Cdc7p-GFP (compare Fig. 1C with Fig. 5B; LF0.4, $P=0.045$ ), confirming earlier analysis by indirect immunofluorescence (Cerutti and Simanis, 1999). Given that the steady state level of Cdc16p at the SPBs is very low from the onset of mitosis, whereas Byr4p persists until metaphase, these data also show that the interdependence of localisation of the GAP proteins does not apply in early mitosis in wild-type cells.

The behaviour of GFP-Byr4p was significantly altered in the plo1-ts 4 mutant. Over $50 \%$ of cells of $\mathrm{LF} \geq 0.6$ still showed symmetric GFP-Byr4p (compare Fig. 5E with Fig. 5G). Quantification of GFP-Byr4p at the SPBs confirmed the decrease in signal intensity at constant spindle length in wildtype (LF0.2 and 0.3) followed by an increase at one SPB (Fig. 5F). In contrast, in plo1-ts 4 the decrease at LF0.2 and LF0.3 and the subsequent asymmetry was less apparent (Fig. 5H; supplementary material Fig. S2B). Cdc16p-GFP could not be analysed in plo1-ts 4 as the tagged protein is thermosensitive. Thus, Plo1p is required to establish the asymmetric localisation of Byr4p in anaphase, consistent with recent studies (Rachfall et al., 2014).

\section{Pom1p inhibits, and Rad24p promotes, the transition to SIN asymmetry}

Phosphorylation of the Sid2p consensus creates a 14-3-3-proteinbinding site (Gupta et al., 2013; Mah et al., 2005). Phosphorylation of Cdc11p by $\mathrm{Sid} 2 \mathrm{p}$ helps promote $\mathrm{Cdc} 7 \mathrm{p}-$ GFP asymmetry in anaphase (Feoktistova et al., 2012), and SPB localisation of the 14-3-3 protein Rad24p in mitosis is dependent on Cdc11p (Mishra et al., 2005). In rad24-D, more cells with long spindles showed a symmetrical distribution of Cdc7p-GFP (Fig. 6B), implicating $\operatorname{Rad} 24 \mathrm{p}$ in promoting Cdc7p-GFP asymmetry during anaphase.

The conserved DYRK family protein kinase Pom1p is required for bipolar growth, septum positioning and is also implicated in mitotic commitment (Bähler and Pringle, 1998; Martin and Berthelot-Grosjean, 2009; Moseley et al., 2009). The onset of
Cdc7p-GFP asymmetry occurred earlier in pom1-D cells (Fig. 6C; $P=0.01$ for LF0.4, $P=0.05$ for LF0.5). Pom $1 p$ associates with the plasma membrane to form a cortical gradient that emanates from the cell tips (Bähler and Pringle, 1998; Hachet et al., 2011), and gradient formation requires the scaffold protein Wsh3p/Tea4p (Hachet et al., 2011). Analysis of Cdc7p-GFP in wsh3-D cells revealed no significant difference from wild-type (Fig. 6D) indicating that the effect of Pom1p upon the SIN is not mediated by its cortical gradient.

The effect of cell size and regulators of mitotic commitment upon Cdc7p-GFP asymmetry

Given that plol affects SIN protein behaviour during mitosis, we studied the effect of the gain-of-function cut12-s11 mutant, which promotes premature recruitment of Plolp to the SPB in interphase (Bridge et al., 1998; Grallert and Hagan, 2002). The distribution of Cdc7p-GFP was not altered significantly in cut12-s11 cells (Fig. 6E), no association of Cdc7p-GFP was seen with interphase SPBs and the initial asymmetric loading of Cdc7p was also unaffected (data not shown).

Fin1p regulates SIN signalling (Grallert et al., 2004), and Sid2p phosphorylates and activates Fin $1 \mathrm{p}$ at the G2-M transition (Grallert et al., 2012). However, analysis of Cdc7p in a fin1 mutant that cannot be phosphorylated by $\mathrm{Sid} 2 \mathrm{p}$ revealed no significant difference in the distribution of Cdc7p (Fig. 6F). This indicates that the proposed feedback loop promoting SIN protein asymmetry in anaphase does not require Sid2p to phosphorylate Fin1p.

Previous studies have implicated PP2A in SIN regulation (Alcaide-Gavilan et al., 2013; Goyal and Simanis, 2012; Jiang and Hallberg, 2001; Le Goff et al., 2001; Singh et al., 2011); Loss of Ypa2p or Ppa2p function delay the onset of Cdc7p-GFP asymmetry (Goyal and Simanis, 2012). Mutation of the PP2A B' subunit parl rescues some SIN mutants (Jiang and Hallberg, 2001; Le Goff et al., 2001). 50\% of parl-D cells showed symmetric Cdc7p-GFP late in anaphase (compare Fig. $6 \mathrm{H}$ with Fig. 6G), implicating PP2A-Par1p in the establishment of Cdc7p-GFP asymmetry in anaphase B. Loss of the other $\mathrm{B}^{\prime}$ subunit Par2p had no significant effect (Fig. 6I). The CDC14 family phosphatase Flp1p/Clp1p influences mitotic commitment and mitotic exit and affects SIN signalling (Chen et al., 2013; Cueille et al., 2001; Esteban et al., 2004; Mishra et al., 2004; Trautmann et al., 2001; Wolfe and Gould, 2004). However, loss of Clp1p/Flp1p did not alter the Cdc7p-GFP distribution significantly (Fig. 6J).

Previous studies have revealed an intramitotic control in $S$. pombe (Hagan et al., 1990). To investigate whether cell size affected the asymmetric transition of the SIN, Cdc7p-GFP was analysed in wee1-6 and cdc25-22 cells (Russell and Nurse, 1986; Russell and Nurse, 1987). Plotting the intensity of Cdc7p-GFP against spindle length revealed that $>95 \%$ of $9 \mu \mathrm{m}$ spindles showed an asymmetric signal in wild-type cells (supplementary material Fig. S3A). This threshold was reached at $6 \mu \mathrm{m}$ (supplementary material Fig. S3B) in the loss-of-function weel6 mutant, which divides at a reduced size (supplementary material Fig. S3E). The $c d c 25-22$ mutant, is partly defective at $25^{\circ} \mathrm{C}$ (Fantes, 1979), increasing its cell length at division; in these cells, the $>95 \%$ threshold was not reached until spindle lengths of $>18 \mu \mathrm{m}$ (supplementary material Fig. S3C). When the length fraction at which asymmetry was established was considered, in order to account for the length of the cell in which the spindle was present, the profile of Cdc7p-GFP distribution in weel-6 and 

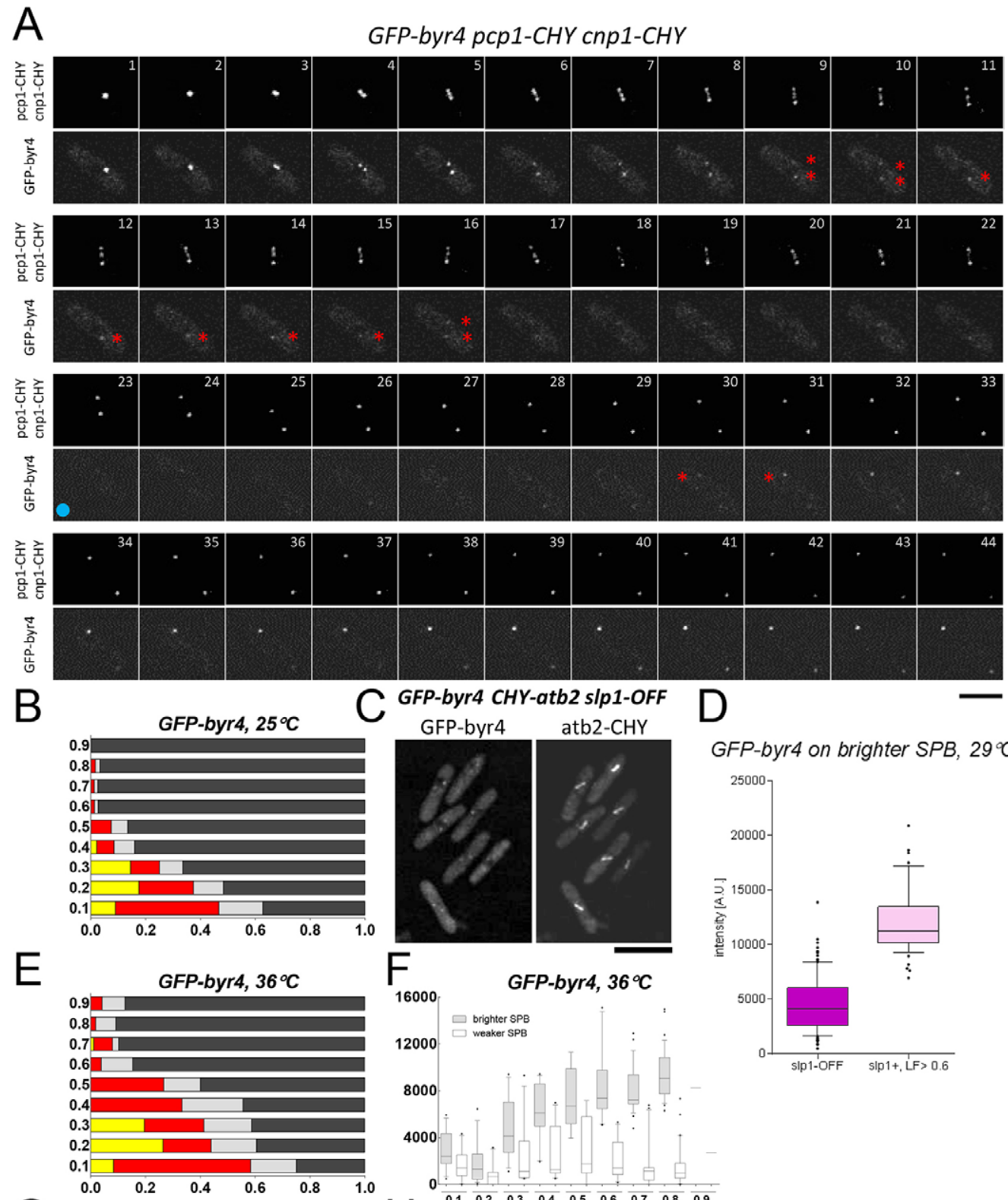

G
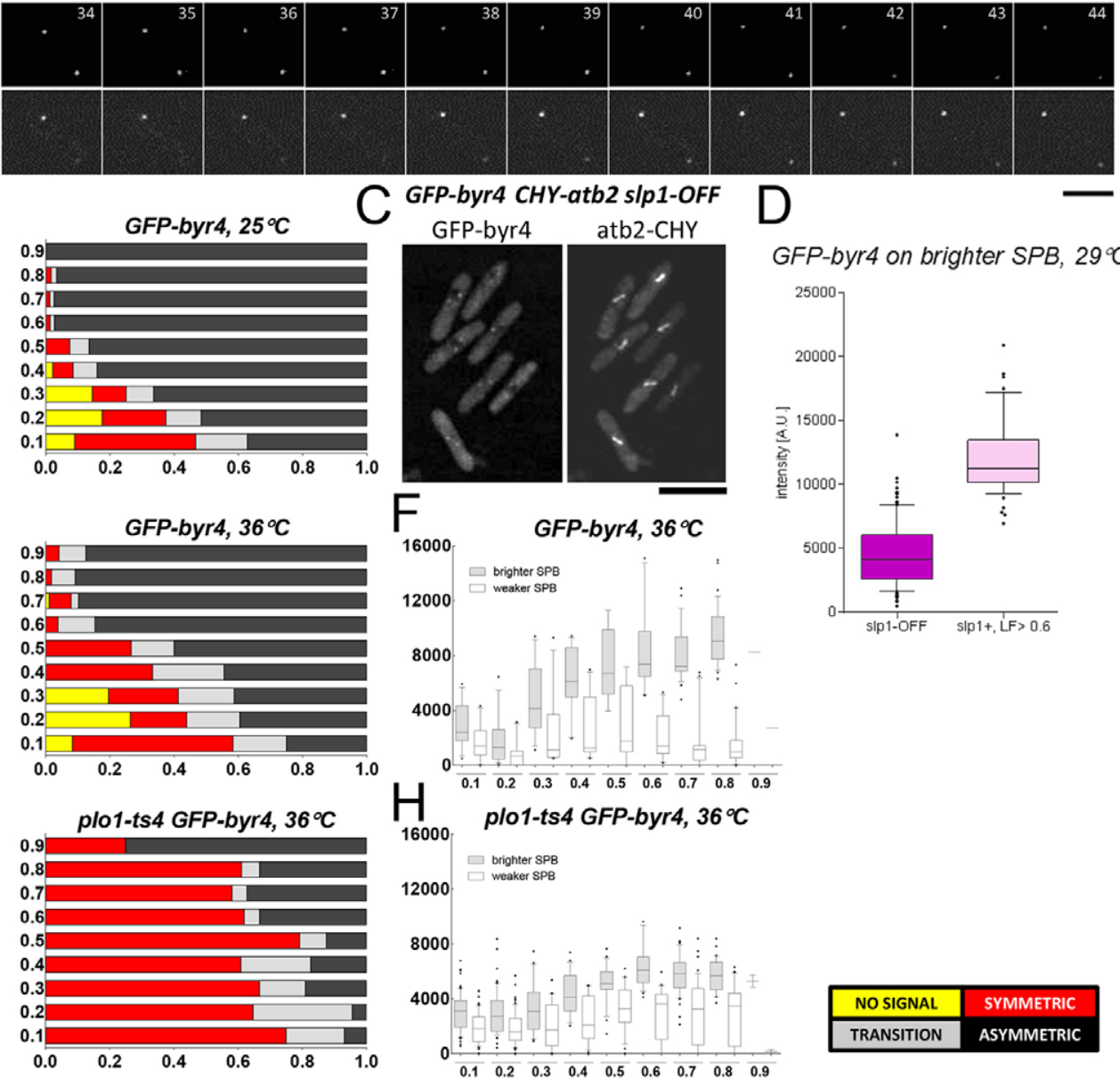

GFP-byr4 CHY-atb2 slp1-OFF D

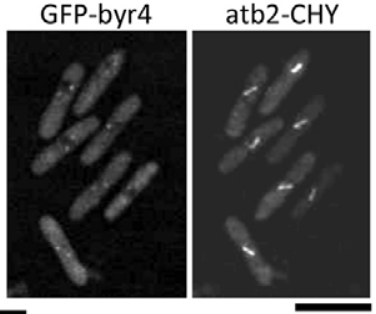

$\mathrm{F}$

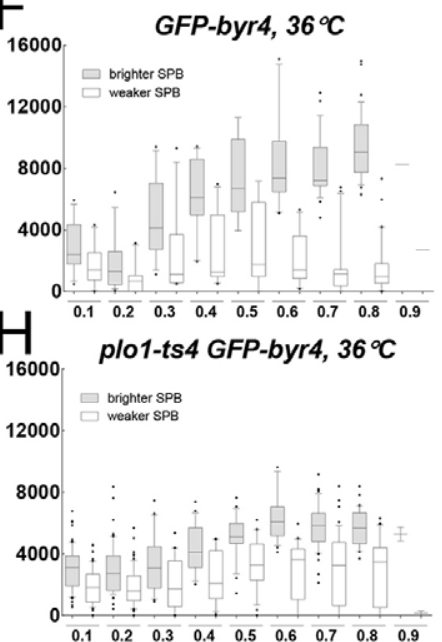

GFP-byr4 on brighter SPB, $29^{\circ} \mathrm{C}$

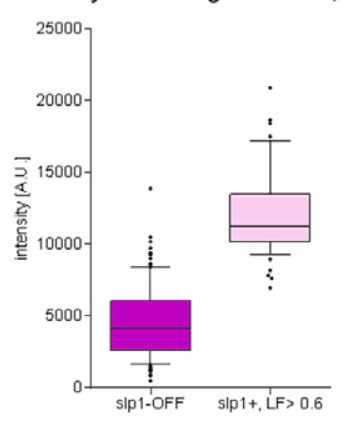

\section{\begin{tabular}{|l|l|}
\hline NO SIGNAL & SYMMETRIC \\
\hline
\end{tabular}} \begin{tabular}{ll}
\hline TRANSITION ASYMMETRIC & ASY \\
\hline
\end{tabular}
Fig. 5. Analysis of GFP-Byr4p in wild-type and mutant cells. (A) The indicated strains were imaged at 1 min intervals. The blue dot

represents anaphase B onset. Note that the GFP-Byr4p signal is initially present on both SPBs, and then decreases in intensity. From frame 9 onwards, the red asterisks indicate SPBs with a GFP-Byr4p signal. A faint signal can also be detected at the other SPB in frames 11 to 15 . The signal is very faint or undetectable from frame 17 to 29; it reappears thereafter on one SPB. Note that the other SPB reacquires a signal from frame 36 onwards. See text for details. $(B, E, G)$ The indicated strains were synchronised and the distribution of GFP-Byr4p on the SPBs was analysed. The graphs are presented as described for Fig. 1C. $(C, D)$ The indicated strain was arrested as described in the Materials and Methods. Note the presence of a weak SPB-associated GFP-Byr4p signal $(C)$; the signal intensity in the arrested cells is compared to that observed at the brightest SPB in anaphase and presented as described in Fig. $2 \mathrm{G}(\mathrm{D})$. $(\mathrm{F}, \mathrm{H})$ The intensity of SPB-associated GFPByr4p is plotted and presented as described in Fig. 2G. Scale bars: $10 \mu \mathrm{m}$. $c d c 25-22$ did not differ significantly from wild-type (compare Fig. $6 \mathrm{~K}$ and Fig. 6L with Fig. 6A). Taken together, these data suggest that the control mechanism over Cdc7p-GFP asymmetry in anaphase scales with cell size.

Elongated cells were also generated by treating wild-type cells with hydroxyurea. Analysis of Cdc7p-GFP after release from the arrest revealed that the threshold for $95 \%$ asymmetry was not reached until a spindle length of $21 \mu \mathrm{m}$ (supplementary material Fig. S3D). Expressing the data in terms of the length fraction, revealed a slight but significant delay in establishment of asymmetry (Fig. 6M LF0.6; $P=0.02$ ). Previous studies have noted mitotic delays after release from hydroxyurea-mediated arrest (Tange and Niwa, 2007), which are likely to result from chromosomal rearrangements following homologousrecombination-mediated replication restart (reviewed by Carr and Lambert, 2013). Whether the DNA structure checkpoint affects SIN protein localisation will be the subject of future studies. 

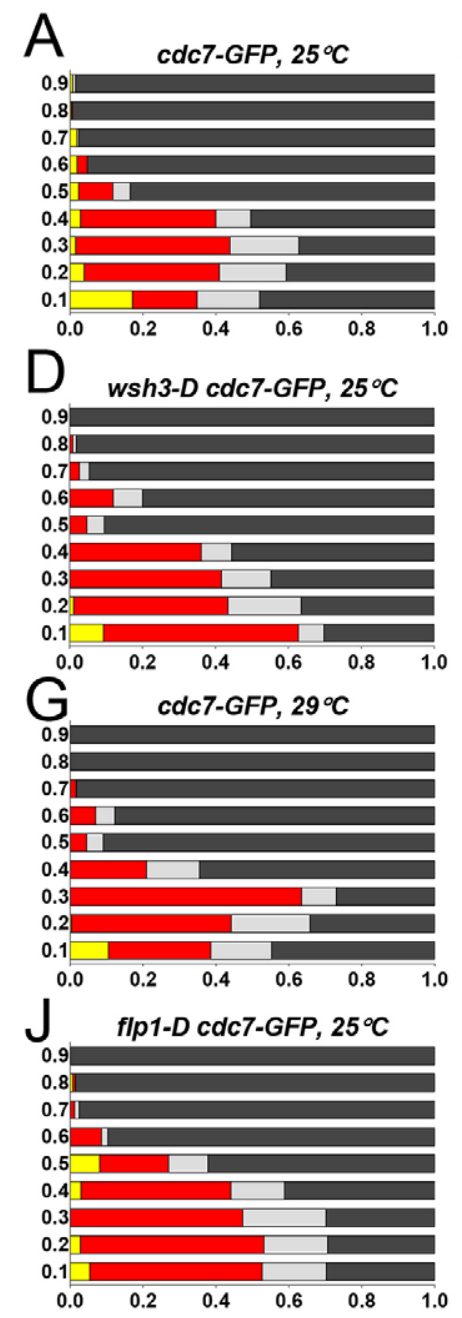

$\mathrm{M}$

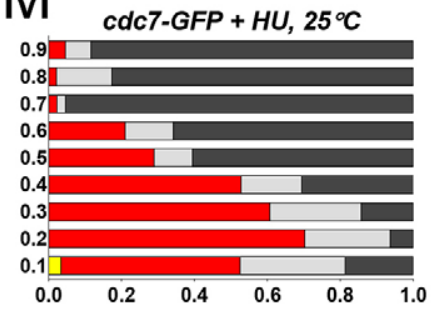

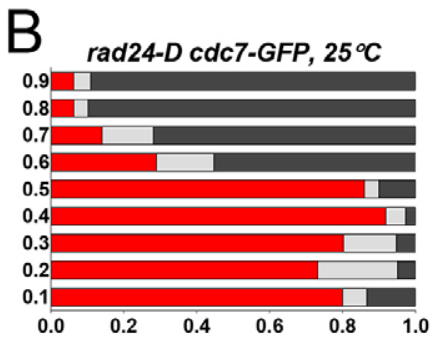
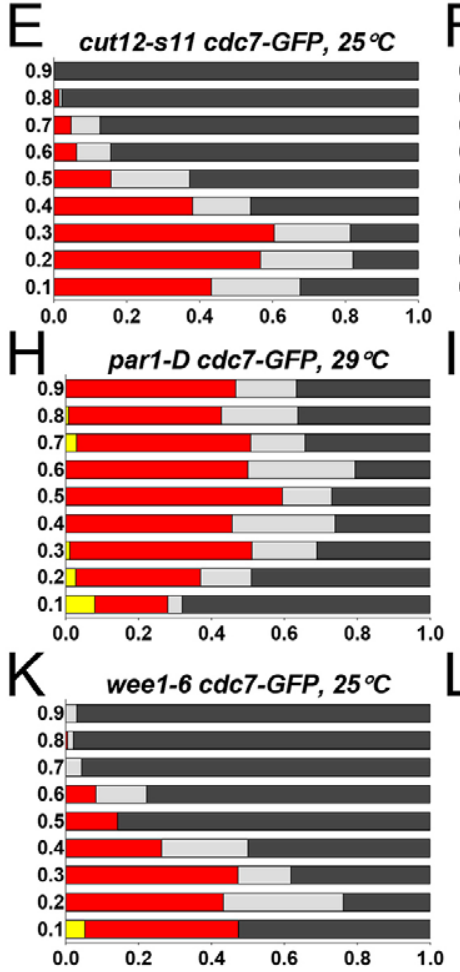

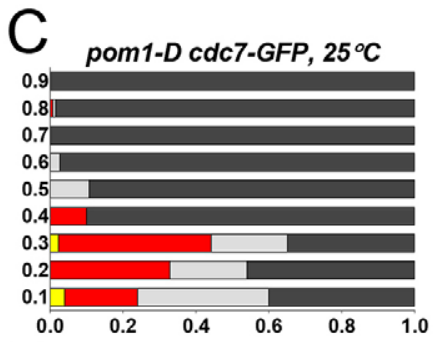

$\mathrm{F}$
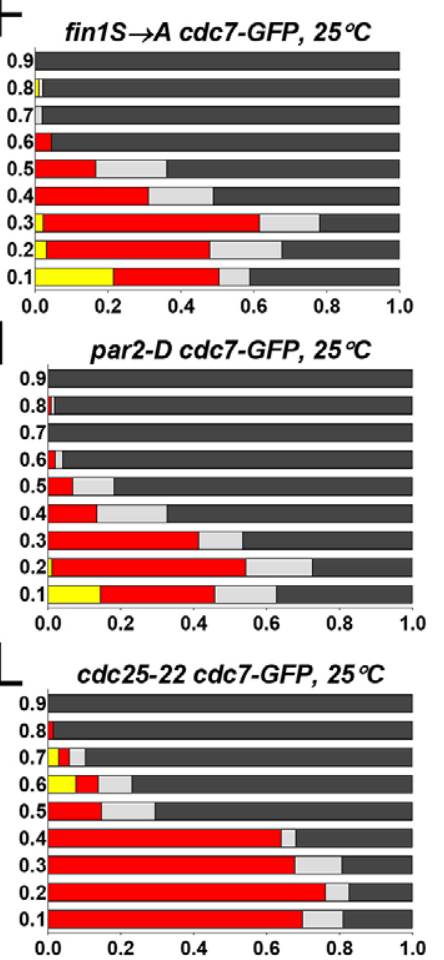

Fig. 6. Analysis of Cdc7p-GFP in wild-type and mutant cells. The intensity of Cdc7p-GFP was analysed in the indicated strains. The graphs are presented as described for Fig. $1 \mathrm{C}$. The $25^{\circ} \mathrm{C}$ wild-type control (A) is duplicated from Fig. 1, for convenience. Par1- $D$ was analysed using asynchronous cultures, as its abnormal shape and asymmetric division preclude efficient synchronisation by elutriation.

Hydroxyurea $(\mathrm{HU})$ indicates that cells were arrested by addition of $12 \mathrm{mM}$ $\mathrm{HU}$ to for $5 \mathrm{~h}$. Cells were analysed in the first mitosis after removal of $\mathrm{HU}$.

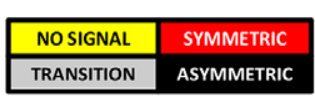

\section{The spindle assembly checkpoint affects Cdc7p-GFP SPB} association in anaphase

Previous studies have suggested that the onset of asymmetry of Cdc7p-GFP in anaphase B is delayed by the presence of unsegregated DNA (Mayer et al., 2006). To investigate this further, we examined mutants in which lagging chromosomes are observed on an extended anaphase spindle.

We first analysed $p c s 1-D$, which ablates the monopolin complex, and generates merotelically attached chromosomes (Gregan et al., 2007). Examination of pcs1-D cdc7-GFP cnplCHY cells revealed that the timing with which Cdc7p-GFP became asymmetric was similar, whether the cells had a lagging kinetochore or not (Fig. 7A,B).

The kinesin-8 protein Klp5p is required for microtubulekinetochore attachment, chromosome congression in metaphase (Garcia et al., 2002a; Garcia et al., 2002b; West et al., 2002) and spindle assembly checkpoint (SAC) silencing (Meadows et al., 2011). In klp5-D cdc7-GFP cnp1-CHY cells ( $\mathrm{LF}>0.5$ ), the Cdc7p-GFP signal was symmetric in cells with long spindles (LF $>0.5$ ) that had lagging kinetochores, and asymmetric in those that did not (Fig. 7C,D).

The apparent contradiction between these mutants might be because the SAC is activated in klp5-D (Garcia et al., 2002b) but not in pcsl-D cells (Gregan et al., 2007). Analysis of klp5-D cdc7-GFP cnp1-CHY pcp1-CHY mad2-D cells showed that the percentage of cells with asymmetric Cdc7p-GFP at a $\mathrm{LF}>0.5$ was similar whether lagging kinetochores were present or not (Fig. 7E,F), and deletion of mad2 did not affect the onset of asymmetry (Fig. $7 \mathrm{G}, \mathrm{H}$ ). These data indicate that the delay in establishing the asymmetry of Cdc7p-GFP in cells that have lagging kinetochores is due to activation of the SAC. 


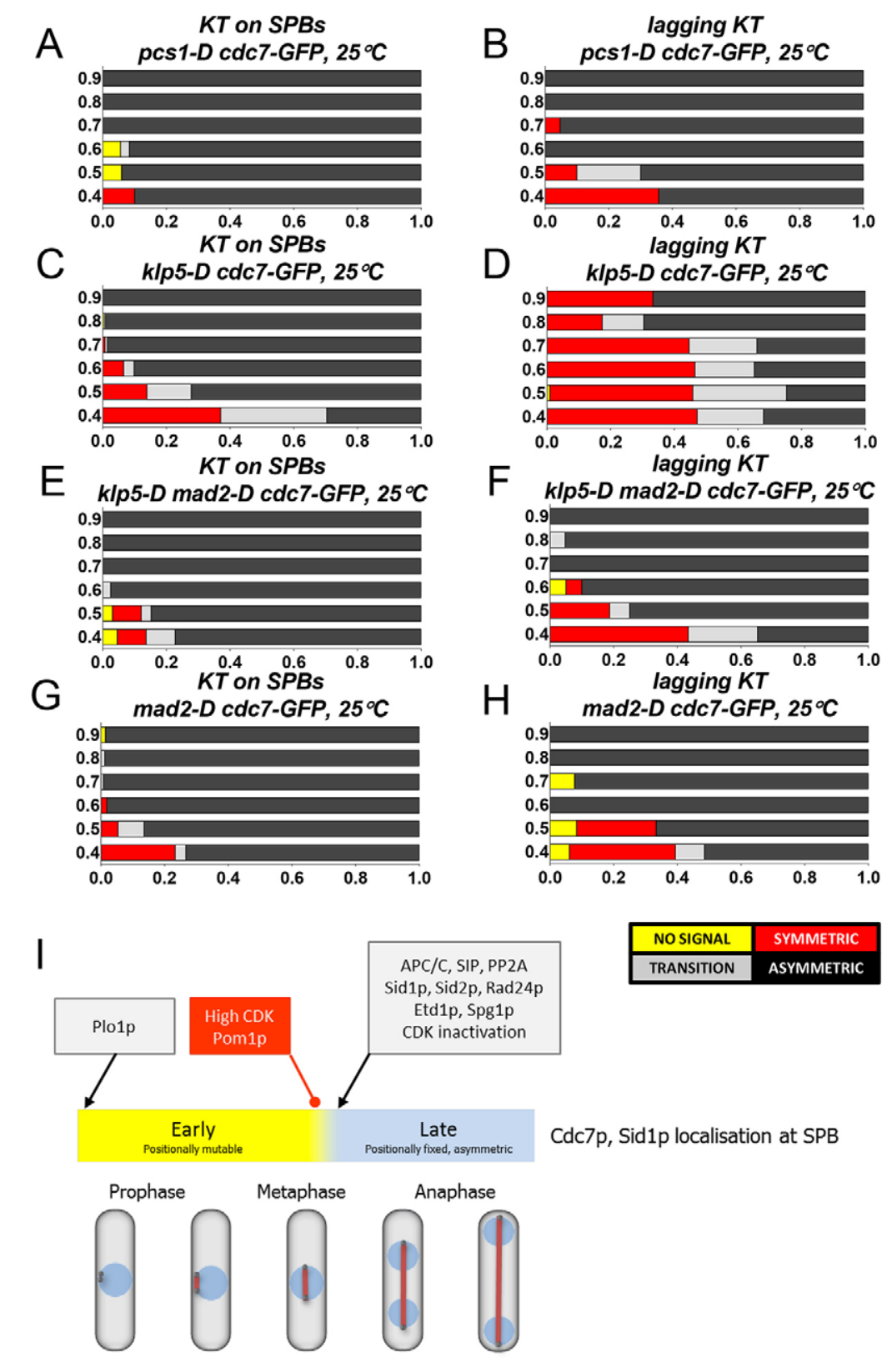

Fig. 7. Analysis of Cdc7p distribution in anaphase in cells with lagging chromosomes and data summary. $(\mathrm{A}-\mathrm{H})$ The strains were analysed at the indicated temperature. The graphs are presented as described for Fig. 1C. For clarity only $L F>0.4$ are shown. (I) Cartoon summarising the two-state model of the SIN. In the early state (yellow), the proteins are positionally mutable, whereas in the late state, their position is fixed and asymmetric. Factors which promote these states (arrow) or inhibit (ball) the transition between them are shown in boxes above. See text for additional details. The lower part shows cartoons of $S$. pombe cells at different stages of mitosis.

\section{DISCUSSION}

\section{A 'two state' representation of the SIN}

This study has revealed several new aspects of SIN regulation. The data point to the existence of two states of the SIN during mitosis, which are referred to herein as 'early' and 'late'. The early state is typified by a mixture of symmetric, transition and asymmetric signals and corresponds to $\mathrm{LF}<0.5$. Many $\mathrm{SIN}$ protein signals are positionally mutable, meaning that the signal intensity varies at a given location during continuous observation. The late state is characterised by the asymmetric association of Cdc7p, Sid1p, Byr4p and Cdc16p with one SPB in $>80 \%$ of cells, which had a positionally stable signal, meaning that it was seen at one principal location; this corresponds to $\mathrm{LF} \geq 0.6$ in wild-type cells. The intensity of the SPB signal is greater in the late than early state. This is summarised in Fig. 7I.

\section{What regulates the early-late transition?}

The data presented here show that the control governing the transition between the states of the SIN scales with cell size and can be altered. The transition is inhibited by an active SAC. Previous studies have shown that Cdc2p inactivation promotes the transition to the asymmetric state of the SIN (Chang et al., 2001; Dischinger et al., 2008; Yamano et al., 1996), suggesting that the SAC effect results from delayed inactivation of Cdc2p.

Deletion of the DYRK family protein kinase pom1, accelerated the transition to the late state of the SIN, implying that Pom $1 p$ inhibits it. Interestingly, mutation of Sid $2 p$ sites on Cdc11p also results in an earlier transition to $\mathrm{Cdc} 7 \mathrm{p}-\mathrm{GFP}$ asymmetry (Feoktistova et al., 2012) and, hence, it will be of interest to determine whether Pom1p regulates SIN asymmetry through Cdc11p. 
Ablation of the 14-3-3 protein $\operatorname{Rad} 24 p$ delayed the onset of asymmetry, although not as substantially as loss of Sid2p, SIP or GAP functions; it is possible that the other 14-3-3 protein $\operatorname{Rad} 25 p$ compensates for the loss of Rad24p. A non-exclusive alternative is that not all the effects of Sid2p are mediated by the creation of 14-3-3-binding sites. After reduction of GAP function (cdc16116), the intensity of the $\mathrm{Cdc} 7 \mathrm{p}-\mathrm{GFP}$ signal reached lateanaphase levels earlier than in wild-type cells, indicating that the GAP proteins also inhibit the transition from the early to the late state.

Deletion of the principal PP2A B' regulatory subunit Par1p delayed the onset of $\mathrm{Cdc} 7 \mathrm{p}$ asymmetry, consistent with previous studies conducted by indirect immunofluorescence (Jiang and Hallberg, 2001). Previous studies have shown that mutation of the regulatory B subunit Pablp does not affect the appearance of Cdc7p-GFP at one SPB in anaphase (Lahoz et al., 2010), suggesting that that PP2A-Parlp is involved in promoting the asymmetry of Cdc7p-GFP in anaphase. Previous studies have implicated other PP2A complexes and regulators in promoting the asymmetry of Cdc7p (Goyal and Simanis, 2012; Singh et al., 2011).

A minor population of cells showed asymmetric Cdc7p signals in the absence of GAP or SIP function. This might indicate that the individual proteins that influence asymmetry cooperate to bring about asymmetry. In this context, it is noteworthy that there is a negative genetic interaction between a SIP-complex-null mutant and cdc16-116 (Singh et al., 2011), and PP2A subunits and cdc16-116 (Goyal and Simanis, 2012; Le Goff et al., 2001).

Previous studies have indicated that Etd1p is required both for maintenance of Cdc7p-GFP at the SPBs in anaphase and for the intensity increase of Cdc7p-GFP in anaphase (Alcaide-Gavilan et al., 2013; Daga et al., 2005; Lahoz et al., 2010). Although these studies differ qualitatively in their conclusions, they both implicate Etd1p in establishing the late state of the SIN. Etd1p interacts with Spg1p in vitro, (García-Cortés and McCollum, 2009), suggesting that they cooperate to establish this state of the SIN (Fig. 7I).

Compromising plol function has multiple effects upon the behaviour of SIN proteins. Association of Cdc7p-GFP with the SPB is abolished in early mitosis, but it then appears at one SPB in anaphase. These data imply that association of Cdc7p-GFP with the SPB in early mitosis is not required for its asymmetric SPB association in anaphase, suggesting that these events are controlled differently and perhaps independently. Reduced Plolp activity resulted in a more asymmetric SPB association of Mob1p-GFP in the early state of the SIN. The penetrance was incomplete, which might reflect differences in SPB age, or the hypomorphic plo1-ts 4 allele. The different effects of plo1-ts 4 upon Mob1p-GFP and Cdc7p-GFP might reflect differences in how SPB association of these two proteins is mediated.

Cdc7p-GFP did not show a significant increase in asymmetric signals at $\mathrm{LF} \geq 0.6$ in $\operatorname{spg} 1-B 8$ cells at $36^{\circ} \mathrm{C}$, retaining a distribution similar to that in early mitosis. The intensity of the Cdc7p-GFP signal was similar with and without Spg1p until LF0.5, after which the signal became brighter in the $\operatorname{spg} 1^{+}$cells, and fainter in the spg1-B8 cells. This is consistent with the idea that establishment and/or maintenance of the late state requires Spg1p.

Previous studies have shown that there is no SPB-associated Cdc7p signal in spg1-B8 cells in the second mitosis at the restrictive temperature (Sohrmann et al., 1998). It was therefore surprising to find that inactivation of $\mathrm{Spg} 1 \mathrm{p}$ did not compromise the association of $\mathrm{Cdc} 7 \mathrm{p}-\mathrm{GFP}$ in the early stages of mitosis in the first cycle after the shift to the restrictive temperature.

Given that $\mathrm{Cdc} 7 \mathrm{p}$ associates directly with the scaffold protein Cdc11p (Feoktistova et al., 2012), as well as Spg1p (Mehta and Gould, 2006; Schmidt et al., 1997), these data could be explained if the association of $\mathrm{Cdc} 7 \mathrm{p}$ with the SPB before anaphase depends principally upon binding to $\mathrm{Cdc} 11 \mathrm{p}$. The subsequent absence of Cdc $7 p$ from the SPBs in the second mitosis could be explained by postulating that Cdc7p cannot reload to the SPB unless the SIN has signalled during the first mitosis. This view is supported by previous studies showing that Byr $4 p$ is not seen on all interphase SPBs in tetranucleate SIN mutant cells (Li et al., 2000), and that functional $\operatorname{Sid} 2 p$ is required to establish SIN asymmetry in anaphase (Feoktistova et al., 2012). The first-cycle block to septation in spg1-B8, and the fact that Spg1-B8p-GFP is not associated with the SPB (A.K. and V.S., unpublished data; Krapp et al., 2008), argue that SIN signalling is not functional. Nonetheless, we cannot formally exclude that $\operatorname{spg} 1-B 8$ is a hypomorphic allele and that the presence of the Cdc7p of the SPB in the first mitosis reflects residual Spg1-B8p function. Future analysis of $\operatorname{spg} 1-D$ will resolve this.

In summary, the 'early' state of the SIN requires Plo1p, but not Spg1p for establishment. In contrast, Spg1p becomes important after LF0.5, when it is required to generate Cdc7p-GFP asymmetry and to maintain a strong asymmetric Cdc $7 \mathrm{p}-\mathrm{GFP}$ signal at the SPB.

Analysis of the $S$. cerevisiae mitotic exit network (MEN) has shown that $\mathrm{CDC} 15 \mathrm{p}(\mathrm{Cdc} 7 \mathrm{p})$ requires inputs from both $\mathrm{CDC} 5 \mathrm{p}$ (Plo1p) and TEM1p (Spg1p) for SPB association in anaphase (Rock and Amon, 2011). Although the SIN and MEN differ in their 'wiring' (reviewed by Simanis, 2003; Weiss, 2012), this points to a conserved role for Spg1p/TEM1p and Plo1p/CDC5p in regulating the SPB association of $\mathrm{Cdc} 7 \mathrm{p}$ family kinases.

\section{Spatial instability of SIN proteins in the early state}

A defining characteristic of the early state is spatial instability of the signals of some SIN proteins. The GFP-Sid1p was unstable in the early stages of mitosis, in the sense that its location varied between images, appearing at one, two or no SPBs, before finally stabilising at the nSPB in anaphase. No significant signal was seen in most slpl-deficient cells, indicating that the APC/ $\mathrm{C}^{\text {Slp1p }}$ must function for GFP-Sid1p to associate stably with the SPB.

Spatial instability of the Cdc7p signal was also observed in early mitosis, and has recently been observed in anaphase in a byr 4 mutant that cannot be phosphorylated by Cdc2p (Rachfall et al., 2014). Interestingly, Mob1p-GFP showed much less variability in the early state than Cdc7p-GFP, GFP-Sid1p or GFP-Byr4p, suggesting that it might be regulated differently.

The reason for the instability of the early SIN is presently unclear; several non-exclusive possibilities can be considered. In $S$. cerevisiae, the MEN effector CDC14p is activated in a twostep process; the FEAR network promotes transient release from the nucleolar RENT complex, priming it for activation. The release becomes definitive following activation of the MEN, and promotes mitotic exit. If the MEN is not activated, CDC14p release is only transient (reviewed by Weiss, 2012). This has some similarity with the unstable SPB association of SIN proteins, particularly GFP-Sid1p. It is therefore possible that the early state of the SIN reflects 'preparatory' events prior to its full activation at the end of anaphase B. Given that the activity of all the core SIN proteins and scaffolds is required for CAR 
assembly, the early state might be a manifestation of this function.

Laser ablation of the nSPB in anaphase results in increased Cdc7p-GFP association with the oSPB, and it has been proposed that this indicates feedback between the SPBs in anaphase, so that each maintains its character with respect to SIN proteins (Magidson et al., 2006). The flickering of the SIN signals earlier in mitosis might reflect the initiation of these feedback loops. It is also possible that the identity of the SPBs with regard to SIN protein association is less well defined (or less respected) in metaphase than in anaphase.

The spatial instability might also reflect 'vetoing' of premature attempts to initiate SIN signalling by mitotic checkpoints. The SAC can be reactivated even after proteolysis of cyclin B begins (Clute and Pines, 1999), so the unstable SIN protein signals might reflect SAC reactivation following transient chromosome detachment from the spindle. The changes may also be produced by the early mitotic casein kinase I (Dma1p)-dependent checkpoint (Johnson et al., 2013).

Regulators of the GTPase Cdc42p show periodic oscillations between the tips of the cell. This is governed by a combination of feedback loops (Das et al., 2012). Given that there is no clear periodicity in the fluctuations GFP-Sid1p signal, it seems unlikely that these mechanisms govern the appearance of GFPSid1p at the SPBs in early mitosis.

\section{Asymmetric SPB association of Cdc7p at mitotic entry}

This study has revealed that the initial SPB association of Cdc7pGFP is asymmetric in $\approx 80 \%$ of cells, biased at $3: 1$ to the nSPB. SPB duplication is conservative, and analysis of Fin1p has suggested that SPBs mature fully over two cell cycles (Grallert et al., 2004). Given that Cdc7p-GFP associates asymmetrically with the SPBs during spindle formation, the choice of SPB might reflect the age of the SPB.

Asymmetric association of Cdc $7 p$ and Sidlp with the nSPB is widely considered to represent 'active' SIN signalling. However, our data indicate that SPB association of Cdc $7 \mathrm{p}-\mathrm{GFP}$ in anaphase is insufficient to promote cytokinesis if Plo1p activity is reduced, even though ectopic SIN activation places spg1 and $c d c 7$ downstream of plo1 (Krapp et al., 2003; Tanaka et al., 2001). This might be because the level of signalling that can be achieved is low compared to constitutive activation of Spg1p in cdc16-116. Alternatively, since the SIN cooperates with Mid1p in CAR assembly (Hachet and Simanis, 2008; Huang et al., 2008), failure of an early mitotic Plolp- and/or Cdc7p-dependent event might prevent cytokinesis.

\section{Is Cdc7p asymmetry mediated by regulation of Byr4p?}

The data presented here also show that GFP-Byr4p disappears transiently from both SPBs as cells progress into anaphase, and then reappears on the oSPB. This might account for the failure to detect Byr4p at SPBs in cells with metaphase-length spindles in some studies (Johnson and Gould, 2011; Li et al., 2000; Rachfall et al., 2014). However, if the APC/C ${ }^{\text {Slplp }}$ is blocked, then GFPByr4p is still seen at the SPBs, although the signal was much less intense than in anaphase, indicating that its removal from the $\mathrm{SPBs}$ requires $\mathrm{APC} / \mathrm{C}^{\mathrm{Slplp}}$ function. This is also consistent with SPB localisation of Byr4p as determined by indirect immunofluorescence in nda3-KM311 arrested cells (Krapp et al., 2003).

Previous studies of SIN proteins in fixed cells revealed the presence of early mitotic cells in which $\mathrm{Cdc} 7 \mathrm{p}$ was detected at both SPBs, whereas Byr4p was present on one SPB (Cerutti and Simanis, 1999). Consistent with this, the GFP-Byr4p signal becomes asymmetric before Cdc $7 \mathrm{p}-\mathrm{GFP}$. The decrease in intensity at the oSPB initiated before any increase was seen at the nSPB. This is consistent with a model in which the initiation of asymmetry in wild-type cells is mediated by regulation of Byr4p, and begins at the oSPB. The transition between the two SIN states requires the action of the $\mathrm{APC} / \mathrm{C}^{\mathrm{Slp} 1 \mathrm{p}}$, although it is presently unclear whether this is due to a direct action of $\mathrm{APC} / \mathrm{C}^{\mathrm{Slp} 1 \mathrm{p}}$ or whether it is due to a downstream event.

GFP-Byr4p remained associated with both SPBs throughout mitosis in plo1-ts4 cells, indicating that the asymmetric distribution of Byr4p to the oSPB requires Plo1p function. This is consistent with data showing that $\mathrm{Cdc} 2 \mathrm{p}$ and Plo1p cooperate to regulate Byr4p localisation (Rachfall et al., 2014) and poses an interesting conundrum - if removal of Byr4p from the SPB is required for association of $\mathrm{Cdc} 7 \mathrm{p}$ with the $\mathrm{SPB}$ in anaphase (Rachfall et al., 2014), then how does Cdc7p associate with the SPBs in plo1-ts4? It is possible that removal of Byr4p from the $\mathrm{SPB}$ is not required for $\mathrm{Cdc} 7 \mathrm{p}$ to associate with it if Plo1p has not made modifications at the SPB earlier in mitosis. Alternatively, because there are $\approx 400$ molecules of Cdc7p-GFP at the nSPB in late mitosis (Wu and Pollard, 2005), it is likely that there are a similar number of signalling scaffold complexes at the SPB. The presence of both Cdc7p-GFP and Byr4p-GFP in these cell populations might therefore reflect heterogeneity of the complexes at the SPBs in the plol-ts 4 mutant.

\section{Summary}

The data presented in this study are consistent with the notion of two, genetically separable, states of the SIN; an 'early' state, which is dependent upon Plolp for its establishment, and a 'late' state, which is dependent upon Etd1p and Spg1p. Future studies will investigate how the transition occurs.

\section{MATERIALS AND METHODS}

Standard techniques were used for the growth and manipulation of fission yeast (Moreno et al., 1991). Unless indicated otherwise, cells were grown in yeast extract (YE) medium. Briefly, cells were grown in liquid medium to a density of $3.5 \times 10^{6}-6 \times 10^{6} \mathrm{cells} / \mathrm{ml}$, and centrifugal elutriation (Beckman JS-5.0 system) was used to isolate G2 cells (Schmidt et al., 1997). Cells were concentrated by filtration to minimise stress. After $1 \mathrm{~h}$ recovery, the first and second mitoses after elutriation were imaged. There was no significant difference between whether the data were collected from cells in the first or second cycle. Data from biological repeats for a given cell type were also found to have no statistical differences, so data from multiple experiments have been pooled to generate the graphs presented for each marker or mutant. The majority of GFP-tagged strains used in this study have been described previously (see Krapp and Simanis, 2014 and references therein). Strains expressing $\operatorname{slpl}^{+}$from the $n m t 41$ promoter were grown in EMM2 minimal medium without thiamine and supplemented with amino acids $(100 \mathrm{mg} / \mathrm{l})$ as required. To repress slpl expression, the cells were incubated for $5 \mathrm{~h}$ in $29^{\circ} \mathrm{C}$ in YE medium (Petrova et al., 2013). For hydroxyurea arrest and release, $12 \mathrm{mM}$ hydroxyurea was added to exponentially growing cells. After $5 \mathrm{~h}$, cells were released from the arrest by washing twice in medium.

\section{Microscopy}

Living cells were imaged using a U-Plan-S-Apo $60 \times$ N.A. 1.42 objective lens mounted on a Perkin-Elmer or an Olympus IX-81 spinning disc confocal microscope. The temperature was maintained using a custombuilt heating system. The presence of a cytoplasmic signal of Cdc7pGFP limits the intensity difference estimations to $\sim 10$-fold, as the signal associated with the oSPB reaches the cytoplasmic background. 


\section{Image analysis and processing}

Images were analysed using RodCellJ (Schmitter et al., 2013). The background was set by averaging the cytoplasmic signal in $>100$ mitotic cells. Only signals that were at more than a $90 \%$ confidence interval were considered significant. For analysis, these background values were subtracted from the intensity values of SPBs. Images were processed in ImageJ and Adobe Photoshop CS6, and assembled in Powerpoint.

\section{Statistical analysis}

The statistical analysis was performed using GraphPad Prism v6.03. For comparison between strains, data from at least two experiments $(n>500$ cells) were pooled and binned for the length fractions. The distribution of ratios of SPB intensities was compared for each length fraction using a two-tailed non-parametric Mann-Whitney test.

\section{Acknowledgements}

We thank Mohan Balasubramanian, Kathy Gould, lain Hagan, Christian Häring, Dan McCollum, and Takashi Toda for strains. We thank Manuela Moraru and three referees for critical comments on the paper, and the EPFL imaging core facility.

\section{Competing interests}

The authors declare no competing or financial interests.

\section{Author contributions}

Experiments were conceived of by P.W. and V.S., and performed by P.W. Data and statistical analysis were performed by P.W., A.C., E.C.D.R, J.R. and I.X. A.K. provided many strains prior to publication and much critical intellectual input. Image analysis software RodCell J was updated and maintained by D.Schmitter, D.Sage and M.U. The paper was written by V.S.

\section{Funding}

This work was funded by a grant from the Swiss National Science Foundation SINERGIA program; and by the Ecole Polytechnique Fédérale de Lausanne (EPFL).

\section{Supplementary material}

Supplementary material available online at

http://jcs.biologists.org/lookup/suppl/doi:10.1242/jcs.160150/-/DC1

\section{References}

Alcaide-Gavilan, M., Lahoz, A., Daga, R. R. and Jimenez, J. (2013). Feedback regulation of SIN by Etd1 and Rho1 in fission yeast. Genetics 196, 455-470.

Alvarez-Tabarés, I., Grallert, A., Ortiz, J. M. and Hagan, I. M. (2007). Schizosaccharomyces pombe protein phosphatase 1 in mitosis, endocytosis and a partnership with Wsh3/Tea4 to control polarised growth. J. Cell Sci. 120, 3589-3601.

Bähler, J. and Pringle, J. R. (1998). Pom1p, a fission yeast protein kinase that provides positional information for both polarized growth and cytokinesis. Genes Dev. 12, 1356-1370.

Bähler, J., Steever, A. B., Wheatley, S., Wang, Y., Pringle, J. R., Gould, K. L. and McCollum, D. (1998). Role of polo kinase and Mid1p in determining the site of cell division in fission yeast. J. Cell Biol. 143, 1603-1616.

Bajpai, A., Feoktistova, A., Chen, J. S., McCollum, D., Sato, M., Carazo-Salas, R. E., Gould, K. L. and Csikász-Nagy, A. (2013). Dynamics of SIN asymmetry establishment. PLOS Comput. Biol. 9, e1003147.

Bridge, A. J., Morphew, M., Bartlett, R. and Hagan, I. M. (1998). The fission yeast SPB component Cut12 links bipolar spindle formation to mitotic control. Genes Dev. 12, 927-942.

Carr, A. M. and Lambert, S. (2013). Replication stress-induced genome instability: the dark side of replication maintenance by homologous recombination. J. Mol. Biol. 425, 4733-4744.

Cerutti, L. and Simanis, V. (1999). Asymmetry of the spindle pole bodies and spg1p GAP segregation during mitosis in fission yeast. J. Cell Sci. 112, 2313-2321.

Chang, L. and Gould, K. L. (2000). Sid4p is required to localize components of the septation initiation pathway to the spindle pole body in fission yeast. Proc. Natl. Acad. Sci. USA 97, 5249-5254.

Chang, L., Morrell, J. L., Feoktistova, A. and Gould, K. L. (2001). Study of cyclin proteolysis in anaphase-promoting complex (APC) mutant cells reveals the requirement for $\mathrm{APC}$ function in the final steps of the fission yeast septation initiation network. Mol. Cell. Biol. 21, 6681-6694.

Chen, J. S., Broadus, M. R., McLean, J. R., Feoktistova, A., Ren, L. and Gould, K. L. (2013). Comprehensive proteomics analysis reveals new substrates and regulators of the fission yeast clp1/cdc14 phosphatase. Mol. Cell. Proteomics 12, 1074-1086.

Clute, P. and Pines, J. (1999). Temporal and spatial control of cyclin B1 destruction in metaphase. Nat. Cell Biol. 1, 82-87.
Cueille, N., Salimova, E., Esteban, V., Blanco, M., Moreno, S., Bueno, A. and Simanis, V. (2001). Flp1, a fission yeast orthologue of the s. cerevisiae CDC14 gene, is not required for cyclin degradation or rum $1 \mathrm{p}$ stabilisation at the end of mitosis. J. Cell Sci. 114, 2649-2664.

Daga, R. R., Lahoz, A., Muñoz, M. J., Moreno, S. and Jimenez, J. (2005). Etd1p is a novel protein that links the SIN cascade with cytokinesis. EMBO J. 24, 2436-2446.

Das, M., Drake, T., Wiley, D. J., Buchwald, P., Vavylonis, D. and Verde, F. (2012). Oscillatory dynamics of Cdc42 GTPase in the control of polarized growth. Science 337, 239-243.

Dischinger, S., Krapp, A., Xie, L., Paulson, J. R. and Simanis, V. (2008). Chemical genetic analysis of the regulatory role of Cdc2p in the $\mathrm{S}$. pombe septation initiation network. J. Cell Sci. 121, 843-853.

Esteban, V., Blanco, M., Cueille, N., Simanis, V., Moreno, S. and Bueno, A. (2004). A role for the Cdc14-family phosphatase Flp1p at the end of the cell cycle in controlling the rapid degradation of the mitotic inducer Cdc25p in fission yeast. J. Cell Sci. 117, 2461-2468.

Fankhauser, C. and Simanis, V. (1993). The Schizosaccharomyces pombe cdc14 gene is required for septum formation and can also inhibit nuclear division. Mol. Biol. Cell 4, 531-539.

Fankhauser, C. and Simanis, V. (1994). The cdc7 protein kinase is a dosage dependent regulator of septum formation in fission yeast. EMBO J. 13, 3011-3019.

Fankhauser, C., Marks, J., Reymond, A. and Simanis, V. (1993). The S. pombe cdc16 gene is required both for maintenance of p34cdc2 kinase activity and regulation of septum formation: a link between mitosis and cytokinesis? EMBO J. 12, 2697-2704.

Fantes, P. (1979). Epistatic gene interactions in the control of division in fission yeast. Nature $279,428-430$.

Feoktistova, A., Morrell-Falvey, J., Chen, J. S., Singh, N. S., Balasubramanian, M. K. and Gould, K. L. (2012). The fission yeast septation initiation network (SIN) kinase, Sid2, is required for SIN asymmetry and regulates the SIN scaffold, Cdc11. Mol. Biol. Cell 23, 1636-1645.

Furge, K. A., Wong, K., Armstrong, J., Balasubramanian, M. and Albright, C. F. (1998). Byr4 and Cdc16 form a two-component GTPase-activating protein for the Spg1 GTPase that controls septation in fission yeast. Curr. Biol. 8, 947-954.

Garcia, M. A., Koonrugsa, N. and Toda, T. (2002a). Spindle-kinetochore attachment requires the combined action of Kin I-like Klp5/6 and Alp14/Dis1MAPs in fission yeast. EMBO J. 21, 6015-6024.

Garcia, M. A., Koonrugsa, N. and Toda, T. (2002b). Two kinesin-like Kin I family proteins in fission yeast regulate the establishment of metaphase and the onset of anaphase A. Curr. Biol. 12, 610-621.

García-Cortés, J. C. and McCollum, D. (2009). Proper timing of cytokinesis is regulated by Schizosaccharomyces pombe Etd1. J. Cell Biol. 186, 739-753.

Goyal, A. and Simanis, V. (2012). Characterization of ypa1 and ypa2, the Schizosaccharomyces pombe orthologs of the peptidyl proyl isomerases that activate PP2A, reveals a role for Ypa2p in the regulation of cytokinesis. Genetics 190, 1235-1250

Goyal, A., Takaine, M., Simanis, V. and Nakano, K. (2011). Dividing the spoils of growth and the cell cycle: The fission yeast as a model for the study of cytokinesis. Cytoskeleton (Hoboken) 68, 69-88.

Grallert, A. and Hagan, I. M. (2002). Schizosaccharomyces pombe NIMA-related kinase, Fin1, regulates spindle formation and an affinity of Polo for the SPB. EMBO J. 21, 3096-3107.

Grallert, A., Krapp, A., Bagley, S., Simanis, V. and Hagan, I. M. (2004). Recruitment of NIMA kinase shows that maturation of the S. pombe spindle-pole body occurs over consecutive cell cycles and reveals a role for NIMA in modulating SIN activity. Genes Dev. 18, 1007-1021.

Grallert, A., Connolly, Y., Smith, D. L., Simanis, V. and Hagan, I. M. (2012). The S. pombe cytokinesis NDR kinase Sid2 activates Fin1 NIMA kinase to control mitotic commitment through Pom1/Wee1. Nat. Cell Biol. 14, 738-745.

Grallert, A., Patel, A., Tallada, V. A., Chan, K. Y., Bagley, S., Krapp, A., Simanis, V. and Hagan, I. M. (2013). Centrosomal MPF triggers the mitotic and morphogenetic switches of fission yeast. Nat. Cell Biol. 15, 88-95

Gregan, J., Riedel, C. G., Pidoux, A. L., Katou, Y., Rumpf, C., Schleiffer, A., Kearsey, S. E., Shirahige, K., Allshire, R. C. and Nasmyth, K. (2007). The kinetochore proteins Pcs1 and Mde4 and heterochromatin are required to prevent merotelic orientation. Curr. Biol. 17, 1190-1200.

Guertin, D. A., Chang, L., Irshad, F., Gould, K. L. and McCollum, D. (2000). The role of the sid $1 p$ kinase and cdc14p in regulating the onset of cytokinesis in fission yeast. EMBO J. 19, 1803-1815.

Gupta, S. and McCollum, D. (2011). Crosstalk between NDR kinase pathways coordinates cell cycle dependent actin rearrangements. Cell Div. 6, 19.

Gupta, S., Mana-Capelli, S., McLean, J. R., Chen, C. T., Ray, S., Gould, K. L. and McCollum, D. (2013). Identification of SIN pathway targets reveals mechanisms of crosstalk between NDR kinase pathways. Curr. Biol. 23, 333-338.

Hachet, O. and Simanis, V. (2008). Mid1p/anillin and the septation initiation network orchestrate contractile ring assembly for cytokinesis. Genes Dev. 22, 3205-3216.

Hachet, O., Berthelot-Grosjean, M., Kokkoris, K., Vincenzetti, V., Moosbrugger, J. and Martin, S. G. (2011). A phosphorylation cycle shapes gradients of the DYRK family kinase Pom1 at the plasma membrane. Cell 145, $1116-1128$

Hagan, I. M. (2008). The spindle pole body plays a key role in controlling mitotic commitment in the fission yeast Schizosaccharomyces pombe. Biochem. Soc. Trans. 36, 1097-1101. 
Hagan, I. M., Riddle, P. N. and Hyams, J. S. (1990). Intramitotic controls in the fission yeast Schizosaccharomyces pombe: the effect of cell size on spindle length and the timing of mitotic events. J. Cell Biol. 110, 1617-1621.

Hou, M. C., Salek, J. and McCollum, D. (2000). Mob1p interacts with the Sid2p kinase and is required for cytokinesis in fission yeast. Curr. Biol. 10, 619-622.

Huang, Y., Yan, H. and Balasubramanian, M. K. (2008). Assembly of normal actomyosin rings in the absence of Mid1p and cortical nodes in fission yeast. J. Cell Biol. 183, 979-988.

Ishiguro, J. (1998). Genetic control of fission yeast cell wall synthesis: the genes involved in wall biogenesis and their interactions in Schizosaccharomyces pombe. Genes Genet. Syst. 73, 181-191.

Jiang, W. and Hallberg, R. L. (2001). Correct regulation of the septation initiation network in Schizosaccharomyces pombe requires the activities of par1 and par2. Genetics 158, 1413-1429.

Johnson, A. E. and Gould, K. L. (2011). Dma1 ubiquitinates the SIN scaffold, Sid4, to impede the mitotic localization of Plo1 kinase. EMBO J. 30, 341-354.

Johnson, A. E., McCollum, D. and Gould, K. L. (2012). Polar opposites: finetuning cytokinesis through SIN asymmetry. Cytoskeleton (Hoboken) 69, 686-699.

Johnson, A. E., Chen, J. S. and Gould, K. L. (2013). CK1 is required for a mitotic checkpoint that delays cytokinesis. Curr. Biol. 23, 1920-1926.

Knoblich, J. A. (2010). Asymmetric cell division: recent developments and their implications for tumour biology. Nat. Rev. Mol. Cell Biol. 11, 849-860.

Krapp, A. and Simanis, V. (2014). Dma1-dependent degradation of Septation Initiation Network proteins during meiosis in Schizosaccharomyces pombe. J. Cell Sci. 127, 3149-3161.

Krapp, A., Schmidt, S., Cano, E. and Simanis, V. (2001). S. pombe cdc11p, together with sid4p, provides an anchor for septation initiation network proteins on the spindle pole body. Curr. Biol. 11, 1559-1568.

Krapp, A., Cano, E. and Simanis, V. (2003). Mitotic hyperphosphorylation of the fission yeast SIN scaffold protein cdc11p is regulated by the protein kinase cdc7p. Curr. Biol. 13, 168-172.

Krapp, A., Collin, P., Cano del Rosario, E. and Simanis, V. (2008). Homoeostasis between the GTPase Spg1p and its GAP in the regulation of cytokinesis in S. pombe. J. Cell Sci. 121, 601-608.

Lahoz, A., Alcaide-Gavilán, M., Daga, R. R. and Jimenez, J. (2010). Antagonistic roles of PP2A-Pab1 and Etd1 in the control of cytokinesis in fission yeast. Genetics 186, 1261-1270

Le Goff, X., Buvelot, S., Salimova, E., Guerry, F., Schmidt, S., Cueille, N., Cano, E. and Simanis, V. (2001). The protein phosphatase 2A B'-regulatory subunit par1p is implicated in regulation of the $\mathrm{S}$. pombe septation initiation network. FEBS Lett. 508, 136-142.

Li, C., Furge, K. A., Cheng, Q. C. and Albright, C. F. (2000). Byr4 localizes to spindle-pole bodies in a cell cycle-regulated manner to control Cdc7 localization and septation in fission yeast. J. Biol. Chem. 275, 14381-14387.

Magidson, V., Chang, F. and Khodjakov, A. (2006). Regulation of cytokinesis by spindle-pole bodies. Nat. Cell Biol. 8, 891-893.

Mah, A. S., Elia, A. E., Devgan, G., Ptacek, J., Schutkowski, M., Snyder, M., Yaffe, M. B. and Deshaies, R. J. (2005). Substrate specificity analysis of protein kinase complex Dbf2-Mob1 by peptide library and proteome array screening. BMC Biochem. 6, 22.

Martin, S. G. and Berthelot-Grosjean, M. (2009). Polar gradients of the DYRKfamily kinase Pom 1 couple cell length with the cell cycle. Nature 459, 852-856.

Mayer, C., Filopei, J., Batac, J., Alford, L. and Paluh, J. L. (2006). An extended anaphase signaling pathway for Mad2p includes microtubule organizing center proteins and multiple motor-dependent transitions. Cell Cycle 5, 1456-1463.

Meadows, J. C., Shepperd, L. A., Vanoosthuyse, V., Lancaster, T. C., Sochaj, A. M., Buttrick, G. J., Hardwick, K. G. and Millar, J. B. (2011). Spindle checkpoint silencing requires association of PP1 to both Spc7 and kinesin-8 motors. Dev. Cell 20, 739-750.

Mehta, S. and Gould, K. L. (2006). Identification of functional domains within the septation initiation network kinase, Cdc7. J. Biol. Chem. 281, 9935-9941.

Minet, M., Nurse, P., Thuriaux, P. and Mitchison, J. M. (1979). Uncontrolled septation in a cell division cycle mutant of the fission yeast Schizosaccharomyces pombe. J. Bacteriol. 137, 440-446.

Mishra, M., Karagiannis, J., Trautmann, S., Wang, H., McCollum, D. and Balasubramanian, M. K. (2004). The Clp1p/Flp1p phosphatase ensures completion of cytokinesis in response to minor perturbation of the cell division machinery in Schizosaccharomyces pombe. J. Cell Sci. 117, 3897-3910.

Mishra, M., Karagiannis, J., Sevugan, M., Singh, P. and Balasubramanian, M. K. (2005). The 14-3-3 protein rad24p modulates function of the cdc14p family phosphatase clp1p/flp1p in fission yeast. Curr. Biol. 15, 1376-1383.

Moreno, S., Klar, A. and Nurse, P. (1991). Molecular genetic analysis of fission yeast Schizosaccharomyces pombe. Methods Enzymol. 194, 795-823.

Morrell, J. L., Tomlin, G. C., Rajagopalan, S., Venkatram, S., Feoktistova, A. S., Tasto, J. J., Mehta, S., Jennings, J. L., Link, A., Balasubramanian, M. K. et al. (2004). Sid4p-Cdc11p assembles the septation initiation network and its regulators at the S. pombe SPB. Curr. Biol. 14, 579-584.

Moseley, J. B., Mayeux, A., Paoletti, A. and Nurse, P. (2009). A spatial gradient coordinates cell size and mitotic entry in fission yeast. Nature 459, 857-860.

Nurse, P., Thuriaux, P. and Nasmyth, K. (1976). Genetic control of the cell division cycle in the fission yeast Schizosaccharomyces pombe. Mol. Gen. Genet. 146, 167-178.
Ohkura, H., Hagan, I. M. and Glover, D. M. (1995). The conserved Schizosaccharomyces pombe kinase plo1, required to form a bipolar spindle, the actin ring, and septum, can drive septum formation in G1 and G2 cells. Genes Dev. 9, 1059-1073.

Oliferenko, S., Chew, T. G. and Balasubramanian, M. K. (2009). Positioning cytokinesis. Genes Dev. 23, 660-674.

Petrova, B., Dehler, S., Kruitwagen, T., Hériché, J. K., Miura, K. and Haering, C. H. (2013). Quantitative analysis of chromosome condensation in fission yeast. Mol. Cell. Biol. 33, 984-998.

Pollard, T. D. and Wu, J. Q. (2010). Understanding cytokinesis: lessons from fission yeast. Nat. Rev. Mol. Cell Biol. 11, 149-155.

Proctor, S. A., Minc, N., Boudaoud, A. and Chang, F. (2012). Contributions of turgor pressure, the contractile ring, and septum assembly to forces in cytokinesis in fission yeast. Curr. Biol. 22, 1601-1608.

Rachfall, N., Johnson, A. E., Mehta, S., Chen, J. S. and Gould, K. L. (2014). Cdk1 promotes cytokinesis in fission yeast through activation of the septation initiation network. Mol. Biol. Cell 25, 2250-2259.

Roberts-Galbraith, R. H. and Gould, K. L. (2008). Stepping into the ring: the SIN takes on contractile ring assembly. Genes Dev. 22, 3082-3088.

Rock, J. M. and Amon, A. (2011). Cdc15 integrates Tem1 GTPase-mediated spatial signals with Polo kinase-mediated temporal cues to activate mitotic exit. Genes Dev. 25, 1943-1954.

Rosenberg, J. A., Tomlin, G. C., McDonald, W. H., Snydsman, B. E., Muller, E. G., Yates, J. R., 3rd and Gould, K. L. (2006). Ppc89 links multiple proteins, including the septation initiation network, to the core of the fission yeast spindlepole body. Mol. Biol. Cell 17, 3793-3805.

Russell, P. and Nurse, P. (1986). cdc25+ functions as an inducer in the mitotic control of fission yeast. Cell 45, 145-153.

Russell, P. and Nurse, P. (1987). Negative regulation of mitosis by wee1+, a gene encoding a protein kinase homolog. Cell 49, 559-567.

Salimova, E., Sohrmann, M., Fournier, N. and Simanis, V. (2000). The S. pombe orthologue of the $\mathrm{S}$. cerevisiae mob1 gene is essential and functions in signalling the onset of septum formation. J. Cell Sci. 113, 1695-1704.

Schmidt, S., Sohrmann, M., Hofmann, K., Woollard, A. and Simanis, V. (1997). The Spg1p GTPase is an essential, dosage-dependent inducer of septum formation in Schizosaccharomyces pombe. Genes Dev. 11, 1519-1534.

Schmitter, D., Wachowicz, P., Sage, D., Chasapi, A., Xenarios, I., Simanis, V. and Unser, M. (2013). A 2D/3D image analysis system to track fluorescently labeled structures in rod-shaped cells: application to measure spindle pole asymmetry during mitosis. Cell Div. 8, 6.

Simanis, V. (2003). The mitotic exit and septation initiation networks. J. Cell Sci. 116, 4261-4262.

Singh, N. S., Shao, N., McLean, J. R., Sevugan, M., Ren, L., Chew, T. G., Bimbo, A., Sharma, R., Tang, X., Gould, K. L. et al. (2011). SIN-inhibitory phosphatase complex promotes Cdc11p dephosphorylation and propagates SIN asymmetry in fission yeast. Curr. Biol. 21, 1968-1978.

Sohrmann, M., Schmidt, S., Hagan, I. and Simanis, V. (1998). Asymmetric segregation on spindle poles of the Schizosaccharomyces pombe septuminducing protein kinase Cdc7p. Genes Dev. 12, 84-94.

Song, K., Mach, K. E., Chen, C. Y., Reynolds, T. and Albright, C. F. (1996). A novel suppressor of ras 1 in fission yeast, byr4, is a dosage-dependent inhibitor of cytokinesis. J. Cell Biol. 133, 1307-1319.

Sparks, C. A., Morphew, M. and McCollum, D. (1999). Sid2p, a spindle pole body kinase that regulates the onset of cytokinesis. J. Cell Biol. 146, 777-790.

Tajbakhsh, S., Rocheteau, P. and Le Roux, I. (2009). Asymmetric cell divisions and asymmetric cell fates. Annu. Rev. Cell Dev. Biol. 25, 671-699.

Tanaka, K., Petersen, J., Maclver, F., Mulvihill, D. P., Glover, D. M. and Hagan, I. M. (2001). The role of Plo1 kinase in mitotic commitment and septation in Schizosaccharomyces pombe. EMBO J. 20, 1259-1270.

Tange, Y. and Niwa, O. (2007). Novel mad2 alleles isolated in a Schizosaccharomyces pombe gamma-tubulin mutant are defective in metaphase arrest activity, but remain functional for chromosome stability in unperturbed mitosis. Genetics 175, 1571-1584.

Tomlin, G. C., Morrell, J. L. and Gould, K. L. (2002). The spindle pole body protein Cdc11p links Sid4p to the fission yeast septation initiation network. Mol. Biol. Cell 13, 1203-1214.

Trautmann, S., Wolfe, B. A., Jorgensen, P., Tyers, M., Gould, K. L. and McCollum, D. (2001). Fission yeast Clp1p phosphatase regulates G2/M transition and coordination of cytokinesis with cell cycle progression. Curr. Biol. 11, 931-940.

Weiss, E. L. (2012). Mitotic exit and separation of mother and daughter cells. Genetics 192, 1165-1202.

West, R. R., Malmstrom, T. and Mclntosh, J. R. (2002). Kinesins klp5(+) and klp6(+) are required for normal chromosome movement in mitosis. J. Cell Sci. $115,931-940$.

Wolfe, B. A. and Gould, K. L. (2004). Fission yeast Clp1p phosphatase affects G2/M transition and mitotic exit through Cdc25p inactivation. EMBO J. 23, 919929.

Wu, J. Q. and Pollard, T. D. (2005). Counting cytokinesis proteins globally and locally in fission yeast. Science 310, 310-314.

Yamano, H., Gannon, J. and Hunt, T. (1996). The role of proteolysis in cell cycle progression in Schizosaccharomyces pombe. EMBO J. 15, 5268-5279. 